\title{
Supporting Information for Machine-Learning Coupled Cluster Properties through a Density Tensor Representation
}

Benjamin G. Peyton,${ }^{\dagger}$ Connor Briggs, ${ }^{\dagger}$ Ruhee D'Cunha,${ }^{\dagger}$ Johannes T. Margraf,${ }^{\ddagger}$ and T. Daniel Crawford ${ }^{*, \dagger}$

$\dagger$ Department of Chemistry, Virginia Tech, Blacksburg, VA 24061, USA

$\ddagger$ Chair for Theoretical Chemistry, Technische Universität München, Lichtenbergstrae 4, D-85747 Garching, Germany

E-mail: crawdad@vt.edu 


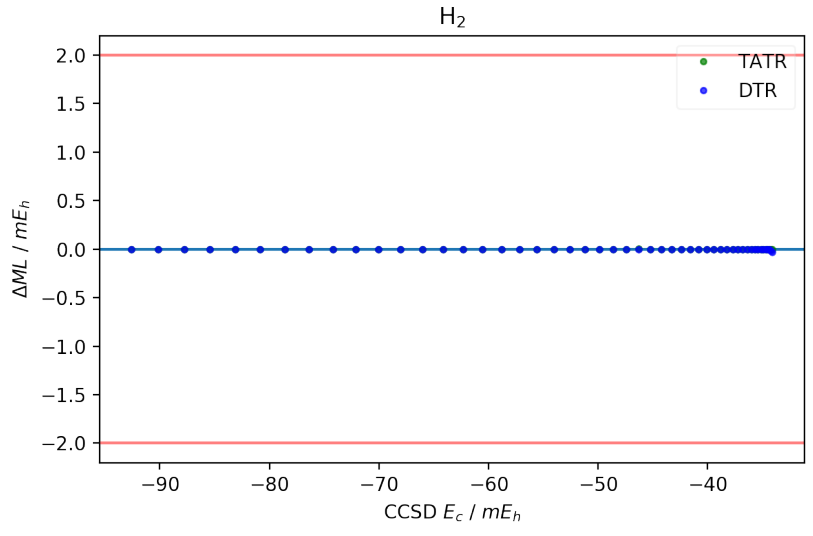

(a)

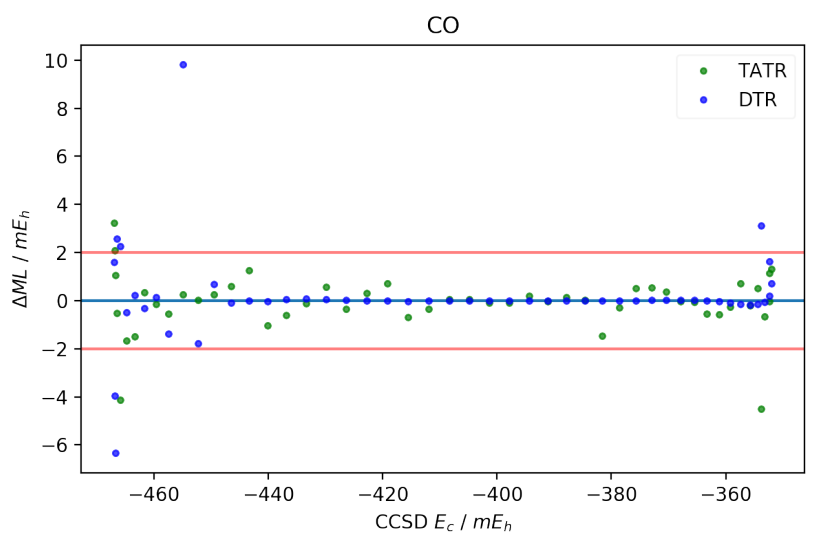

(c)

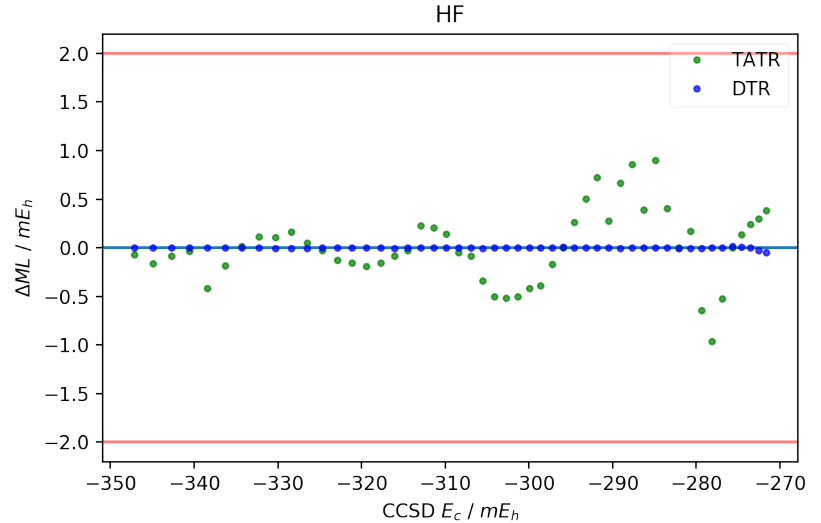

(b)

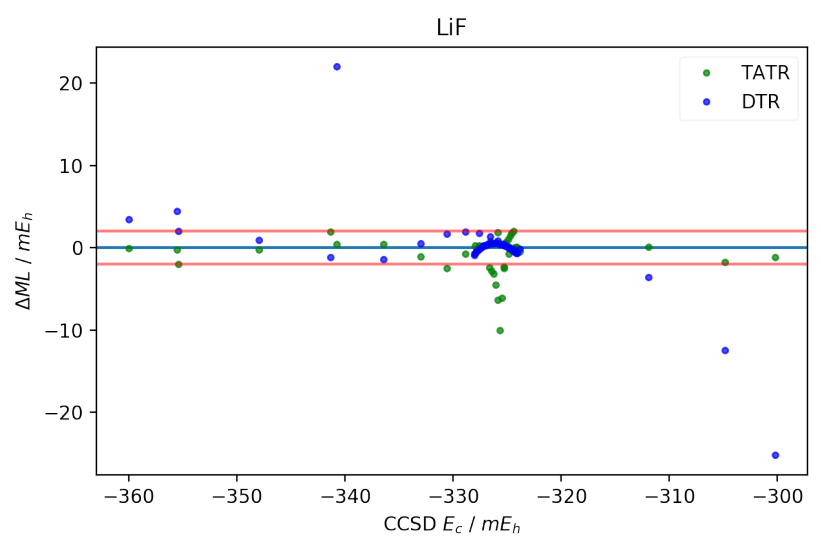

(d)

$\mathrm{N}_{2}$

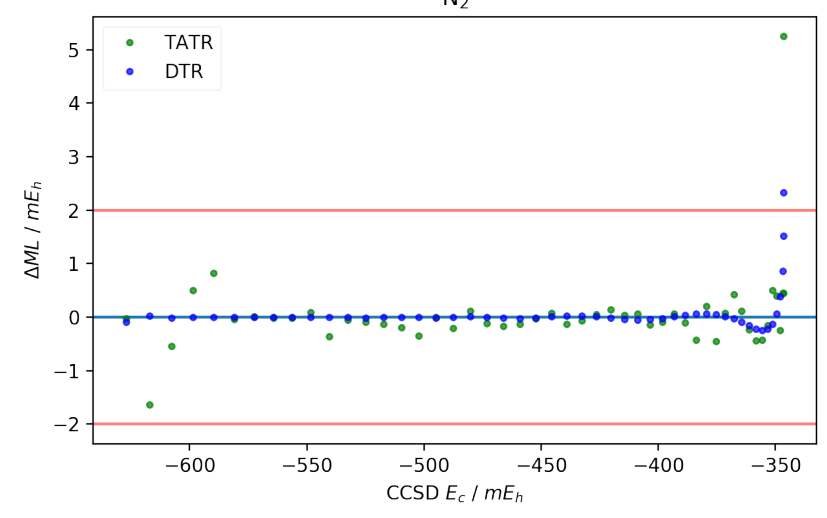

(e)

Figure S1: DTR vs TATR errors in $\mathrm{m} E_{h}$ for diatomic datasets: (a) $\mathrm{H}_{2}$, (b) HF, (c) CO, (d) $\mathrm{LiF}$, and (e) $\mathrm{N}_{2}$. Red lines indicate $2 \mathrm{~m} E_{h}$. 


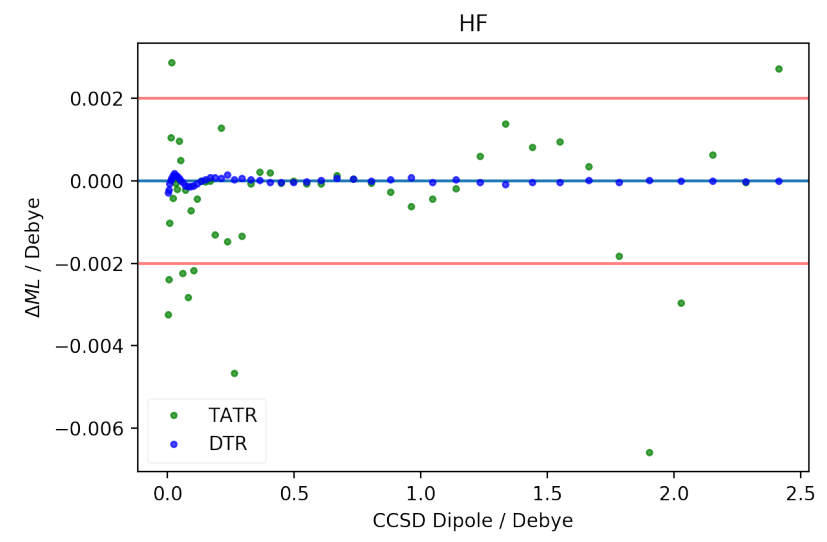

(a)

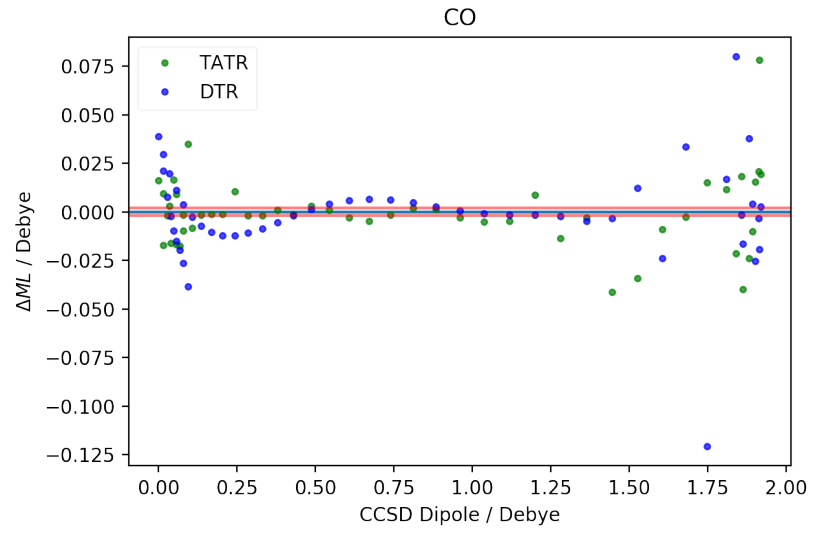

(b)

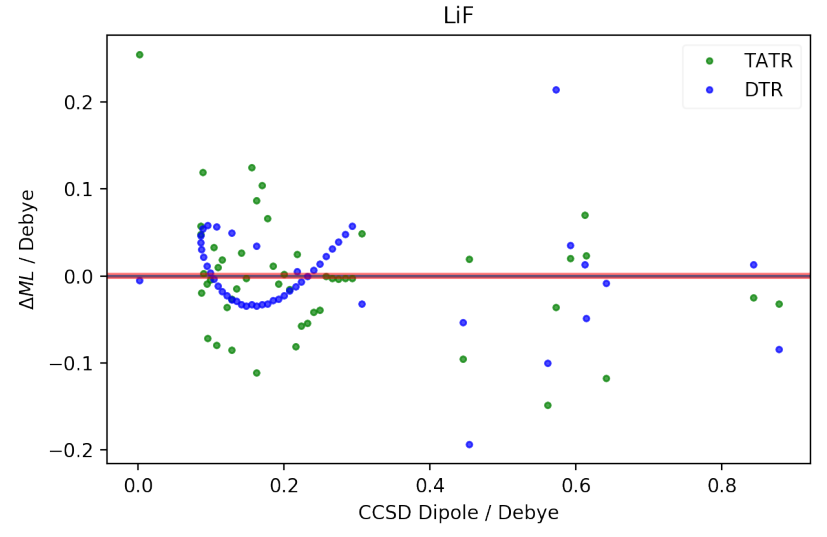

(c)

Figure S2: DTR vs TATR errors in Debye for diatomic datasets: (a) HF, (b) CO, and (c) LiF. Red lines indicate 2 milliDebye. 


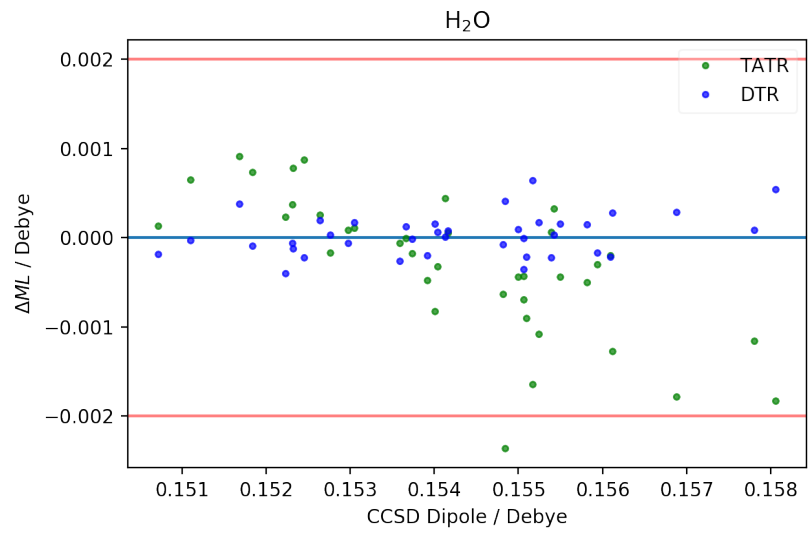

(a)

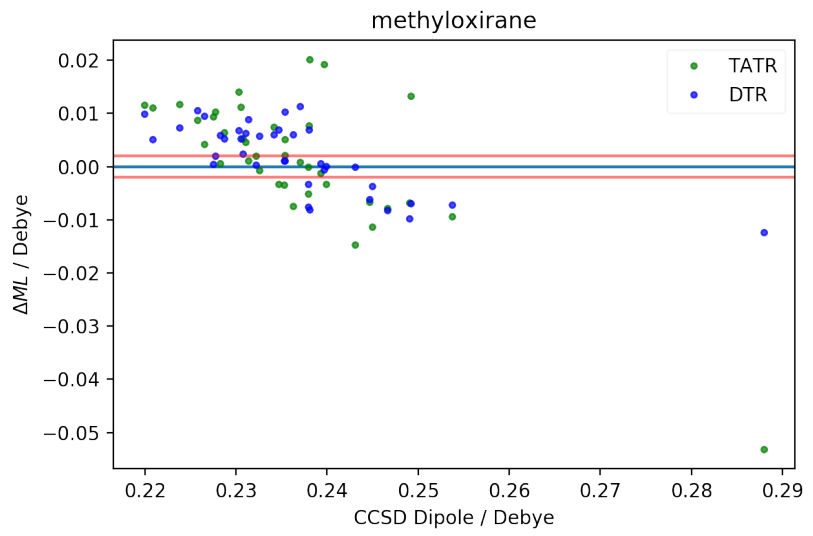

(c)

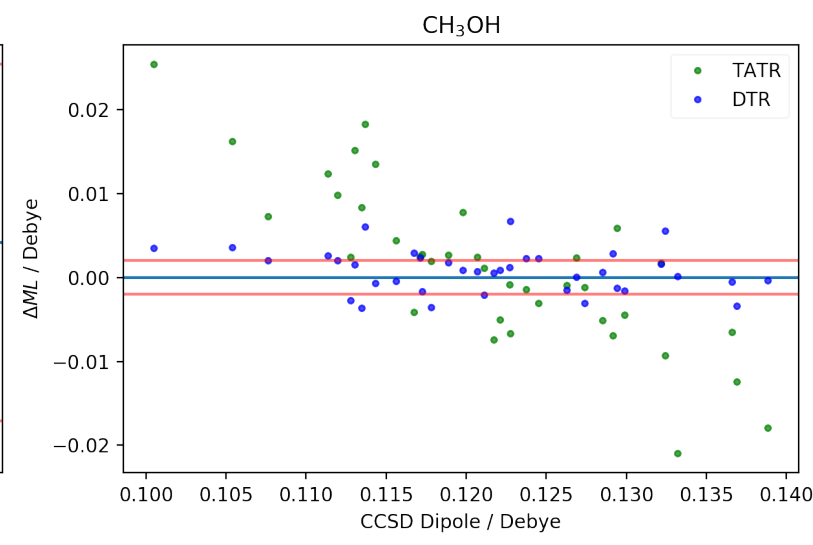

(b)

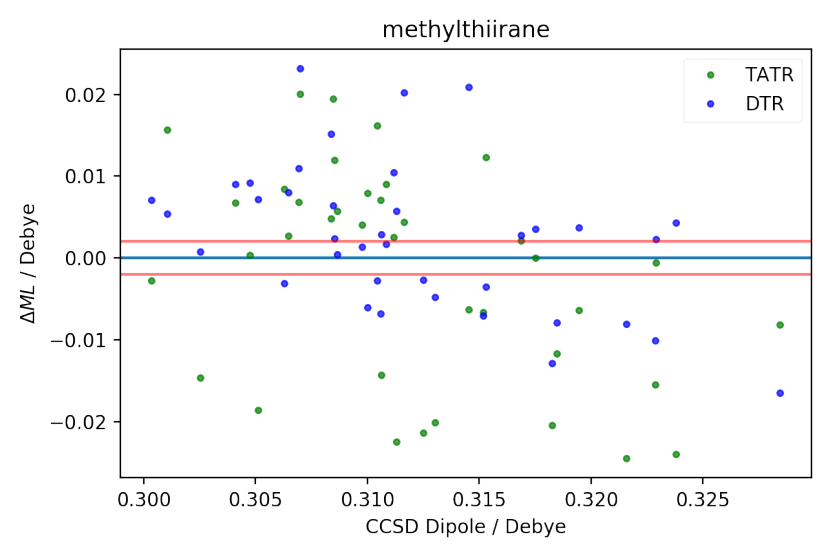

(d)

Figure S3: DTR vs TATR errors in Debye for small molecule datasets: (a) $\mathrm{H}_{2} \mathrm{O}$, (b) $\mathrm{CH}_{3} \mathrm{OH}$, (c) $(S)$-methyloxirane, and (d) $(R)$-methylthiirane. Red lines indicate 2 milliDebye. 


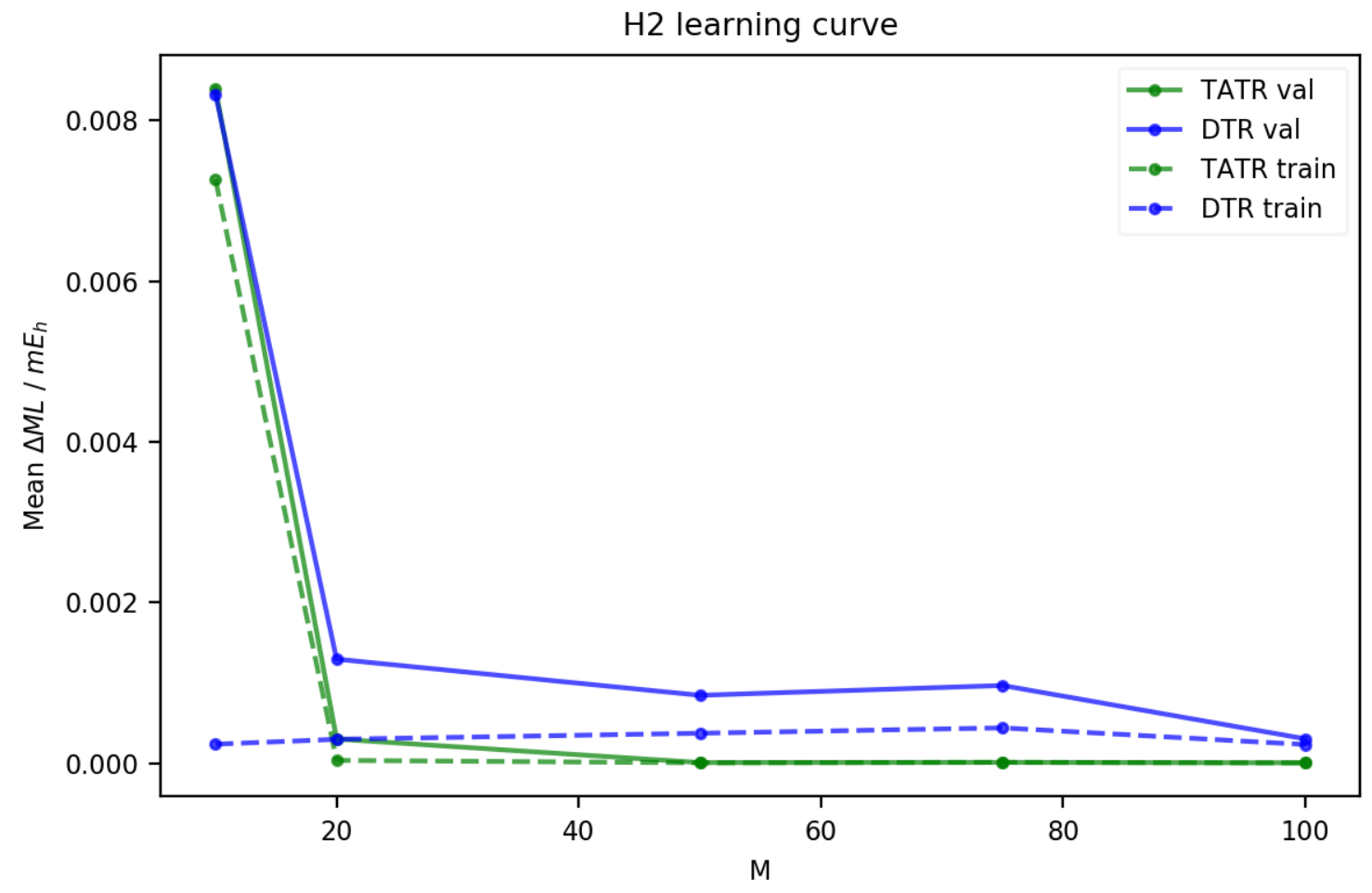

Figure S4: DTR and TATR learning curves for $\mathrm{H}_{2}$ correlation energy.

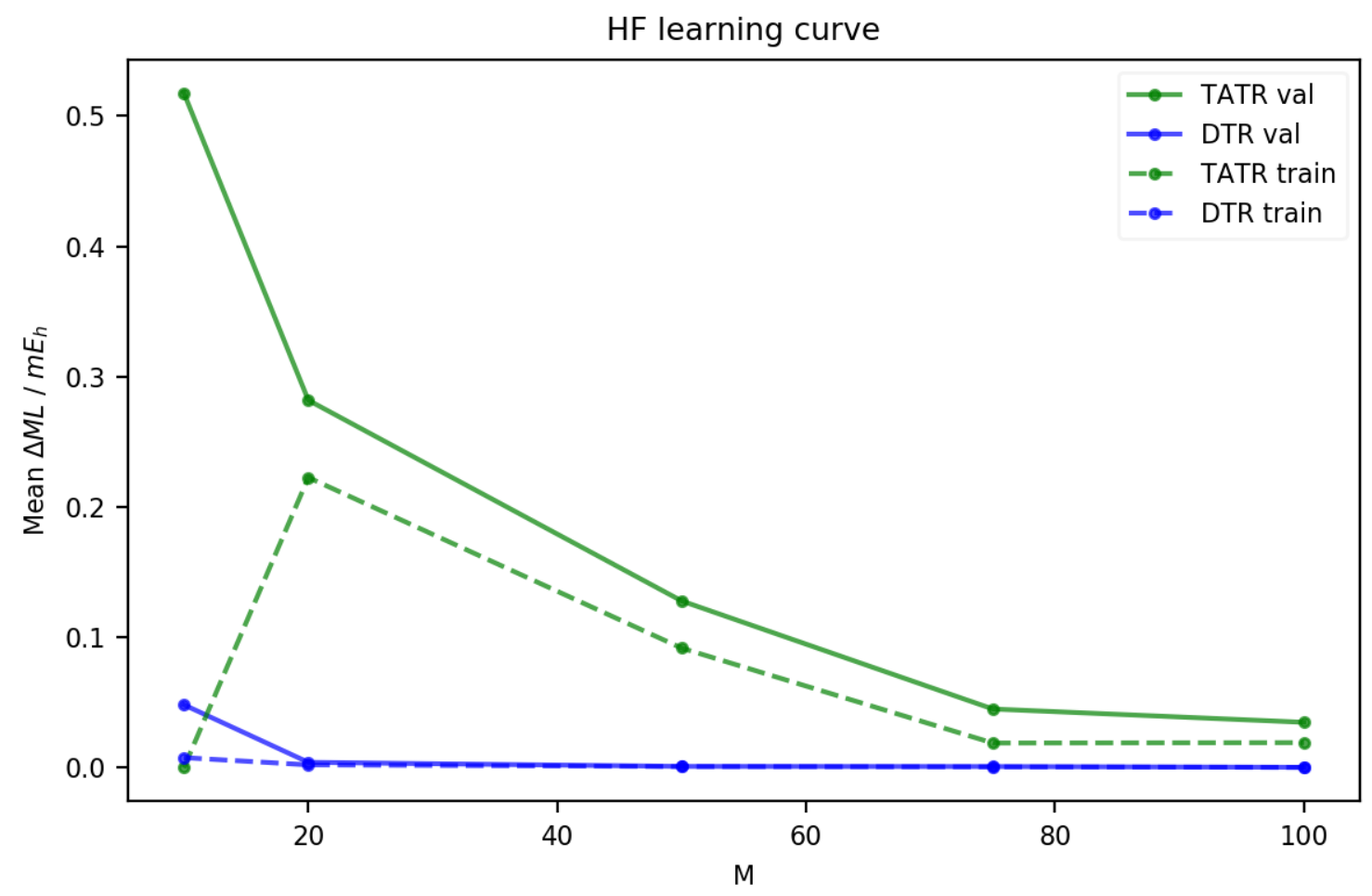

Figure S5: DTR and TATR learning curves for HF correlation energy. 


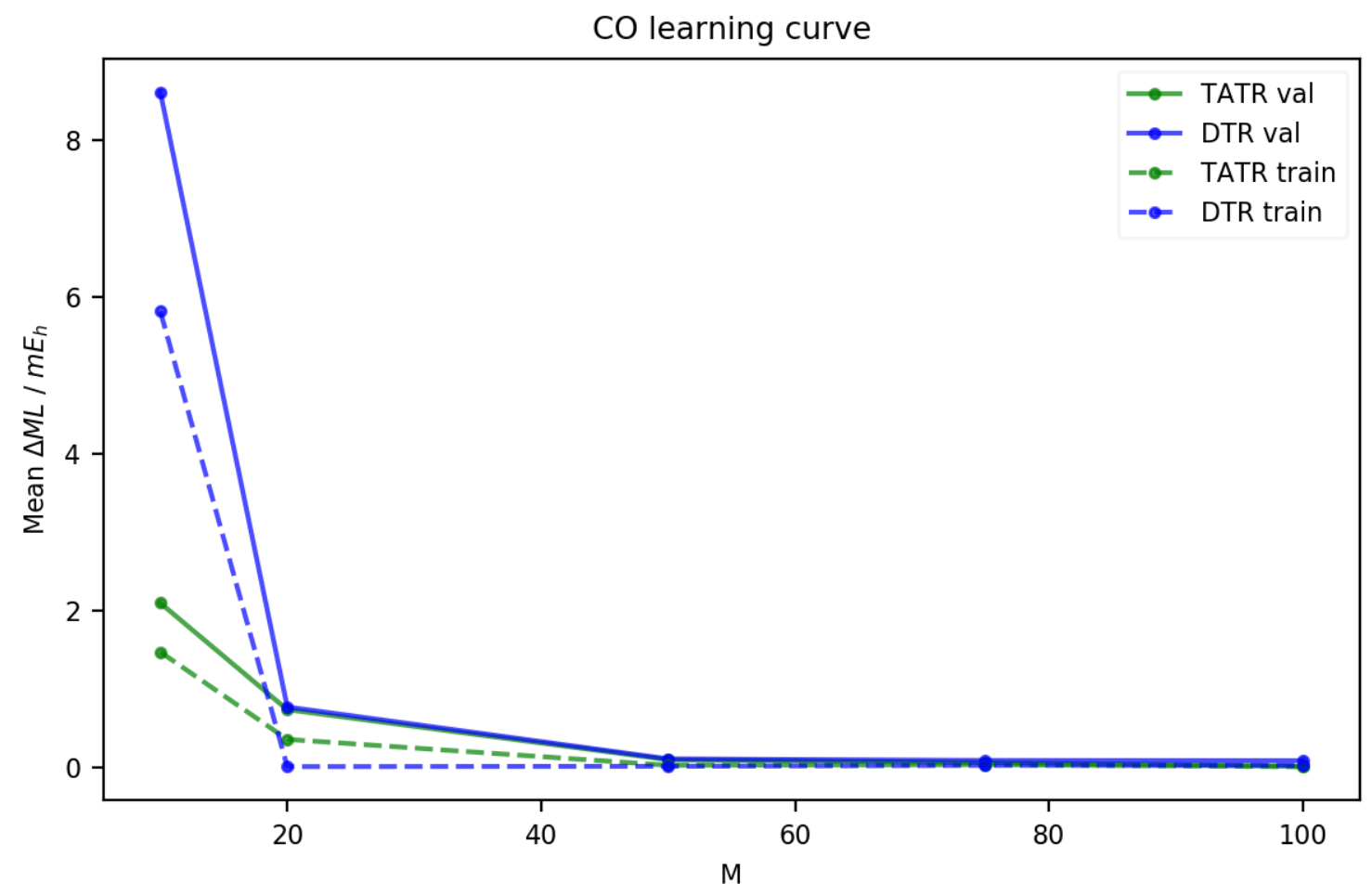

Figure S6: DTR and TATR learning curves for CO correlation energy.

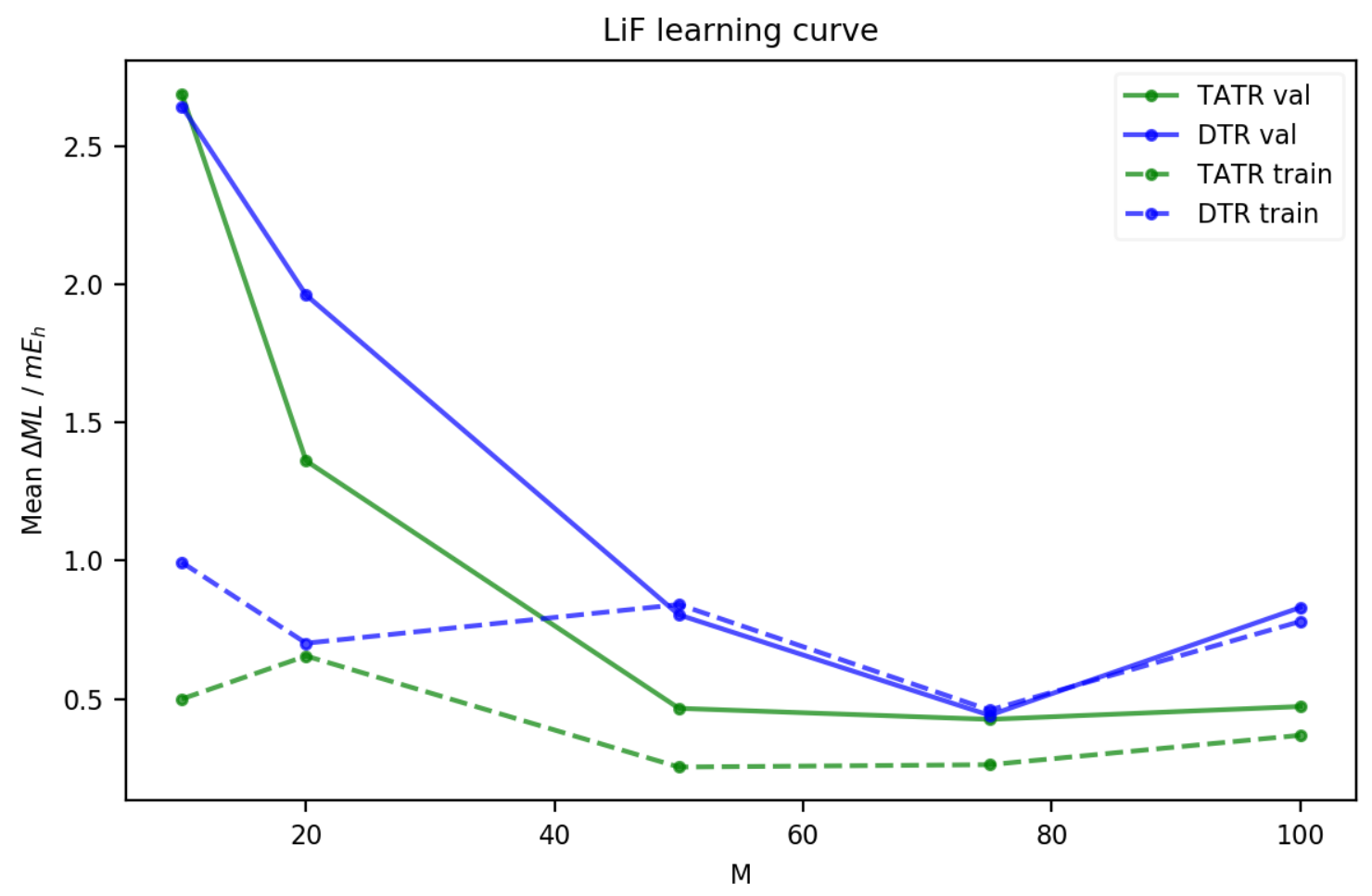

Figure S7: DTR and TATR learning curves for LiF correlation energy. 


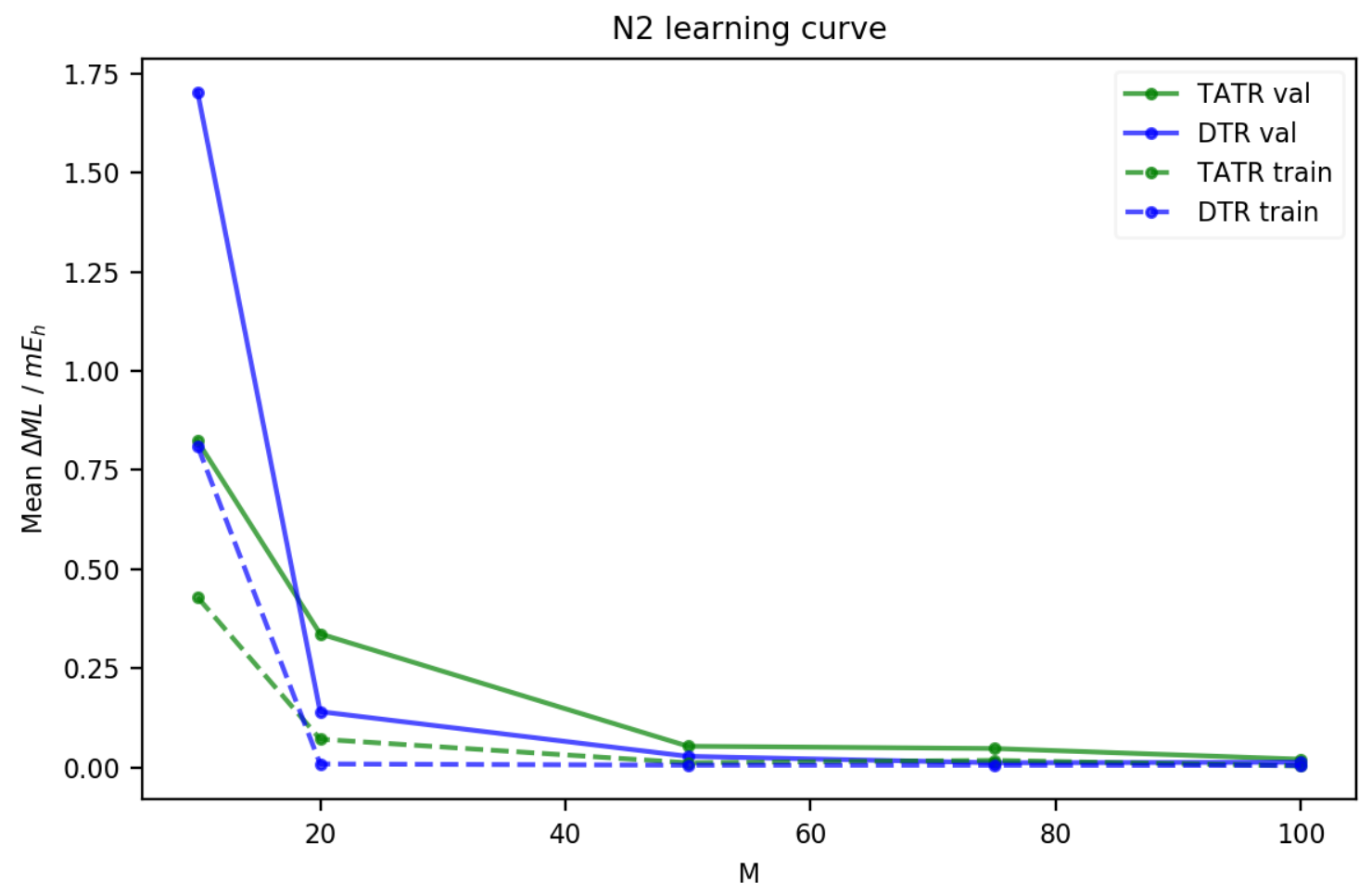

Figure S8: DTR and TATR learning curves for $\mathrm{N}_{2}$ correlation energy.

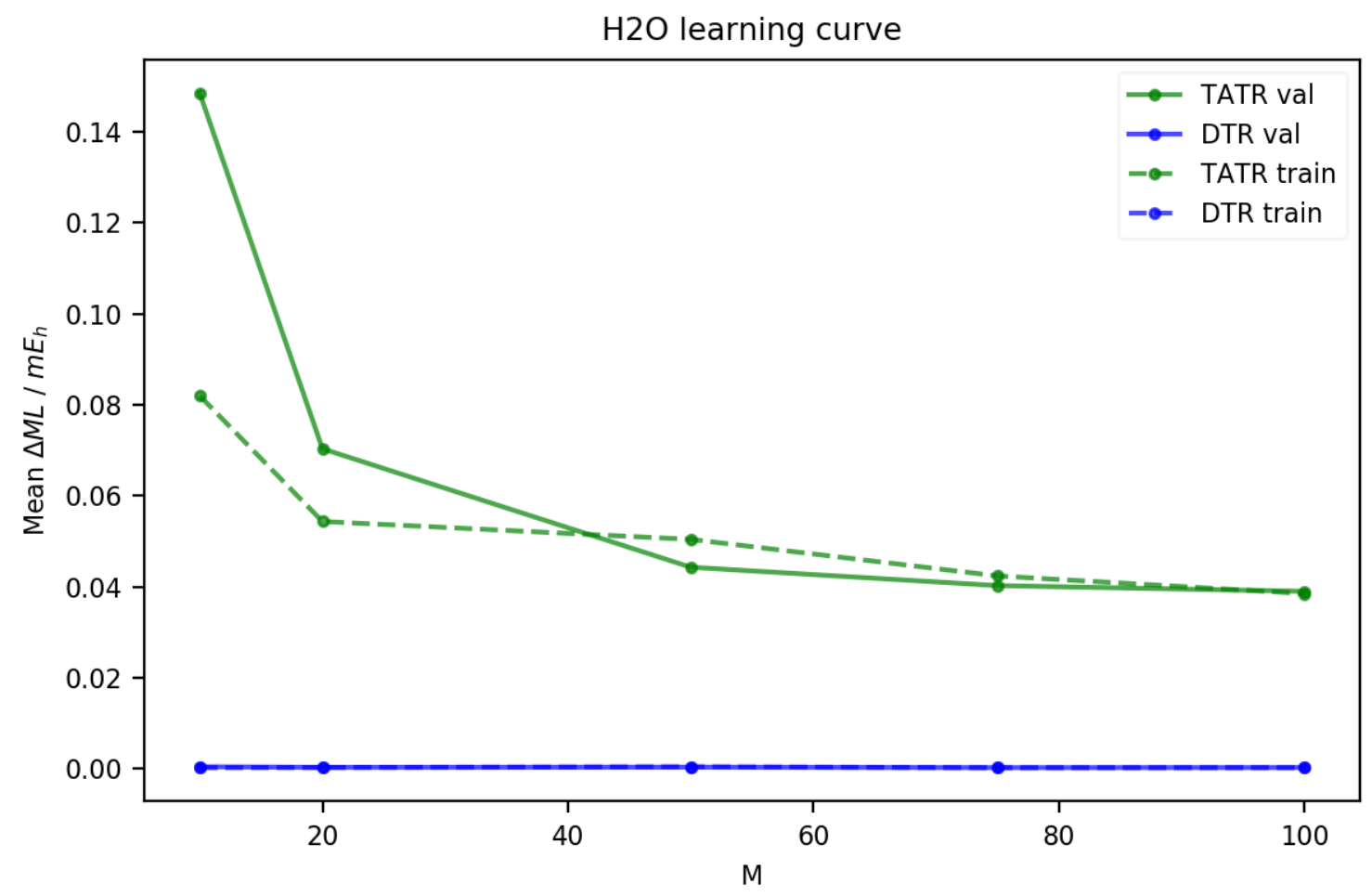

Figure S9: DTR and TATR learning curves for $\mathrm{H}_{2} \mathrm{O}$ correlation energy. 


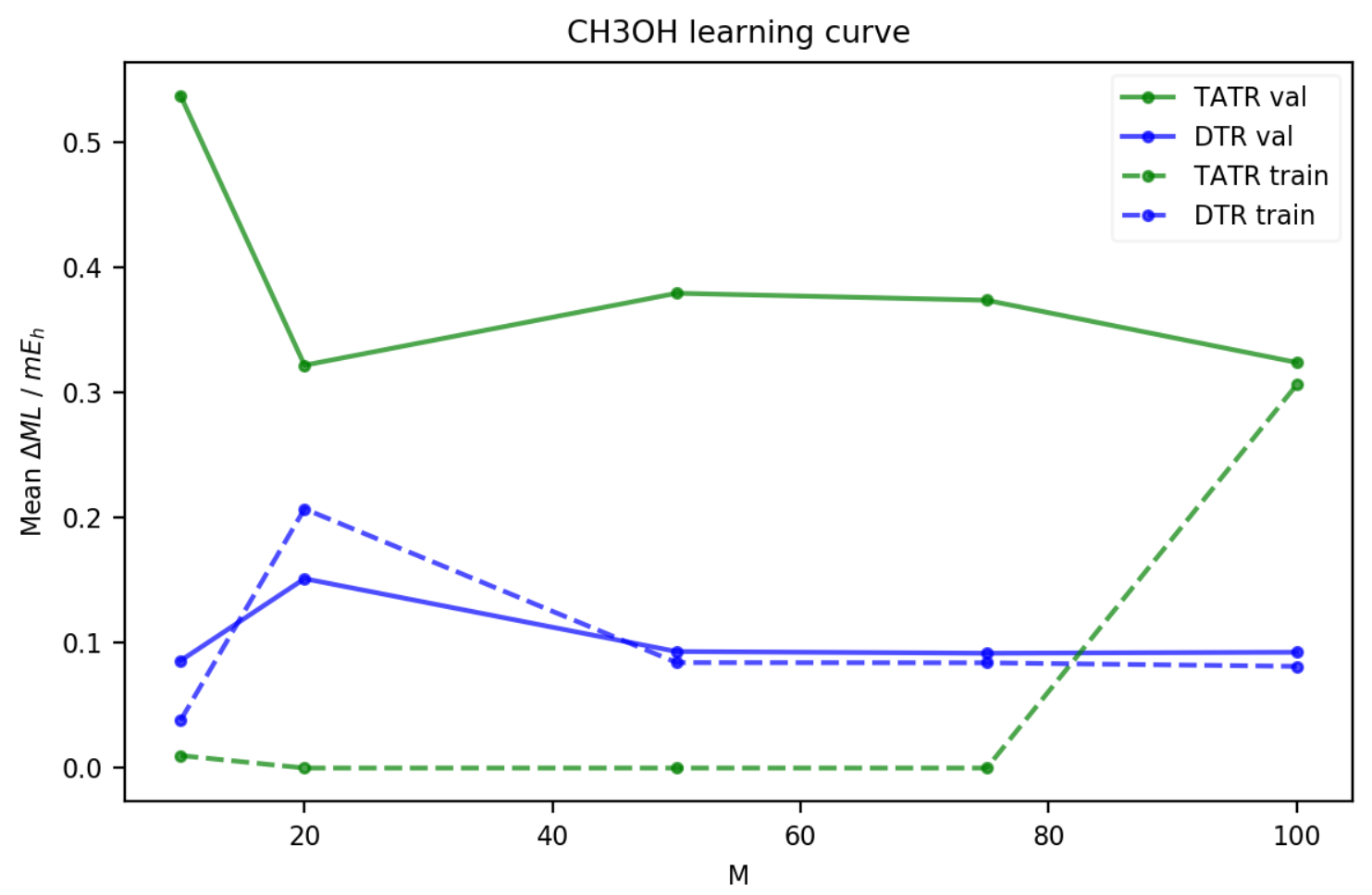

Figure S10: DTR and TATR learning curves for $\mathrm{CH}_{3} \mathrm{OH}$ correlation energy.

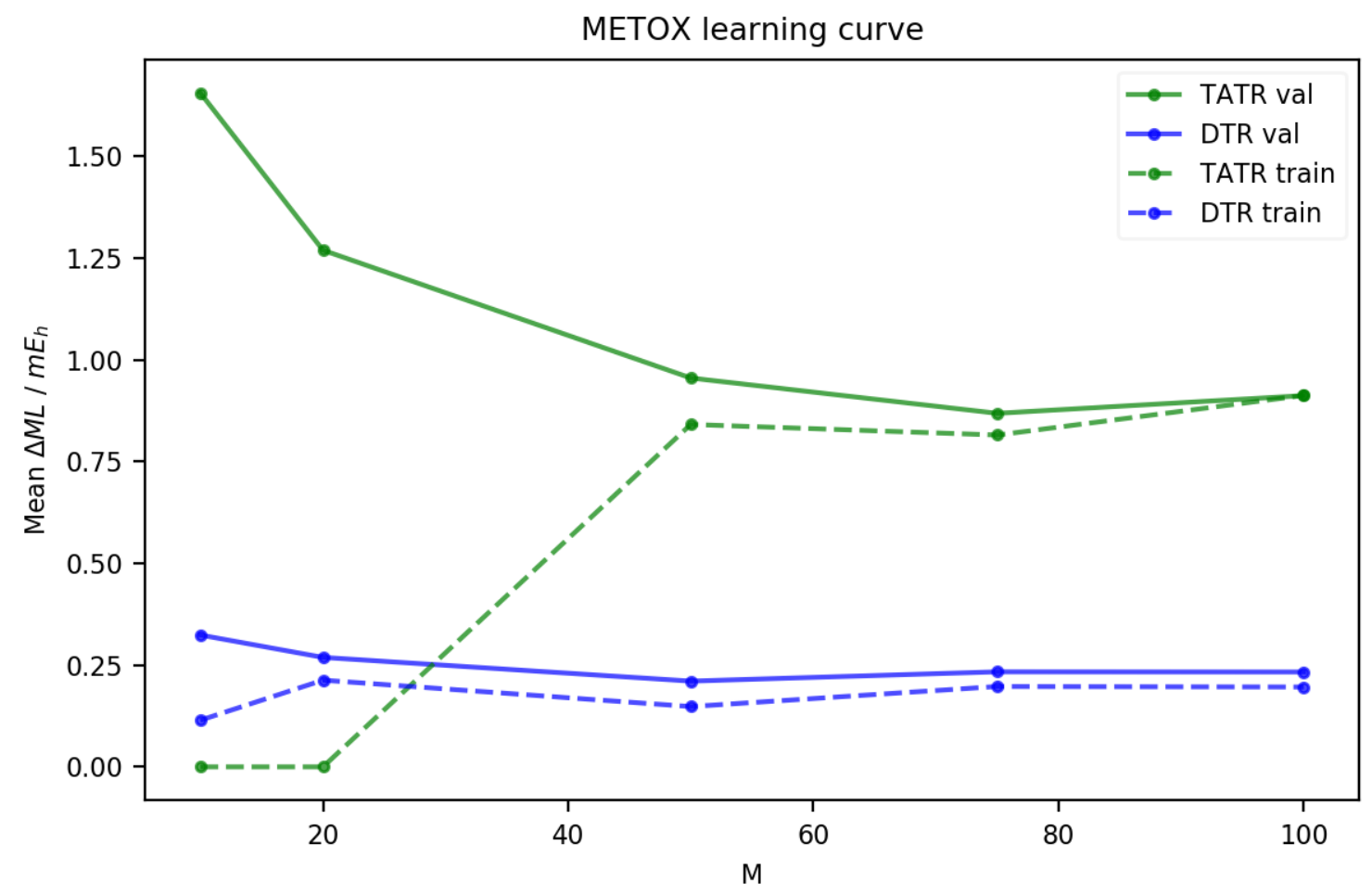

Figure S11: DTR and TATR learning curves for $(S)$-methyloxirane correlation energy. 


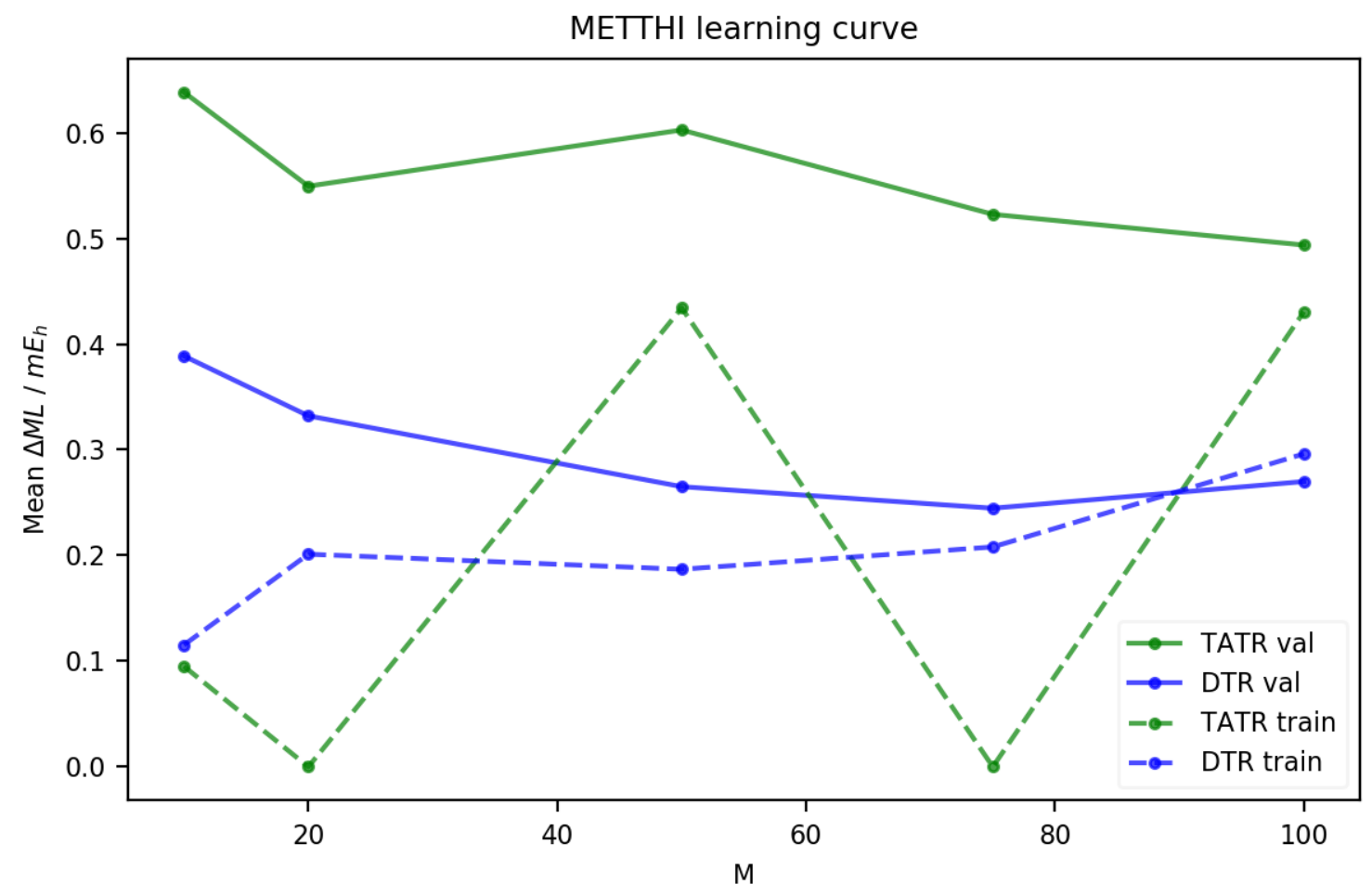

Figure S12: DTR and TATR learning curves for $(R)$-methylthiirane correlation energy.

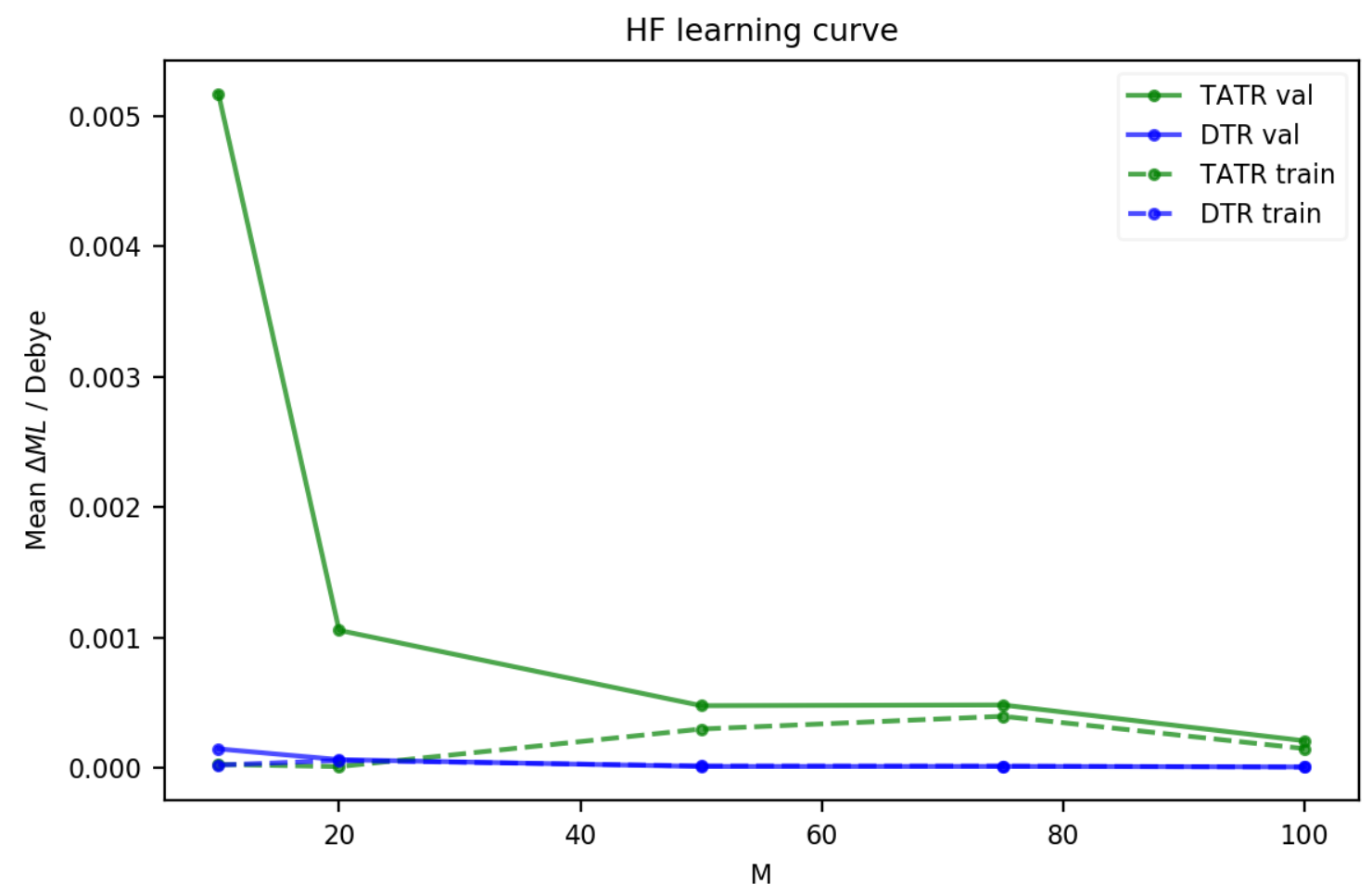

Figure S13: DTR and TATR learning curves for HF correlated dipole. 


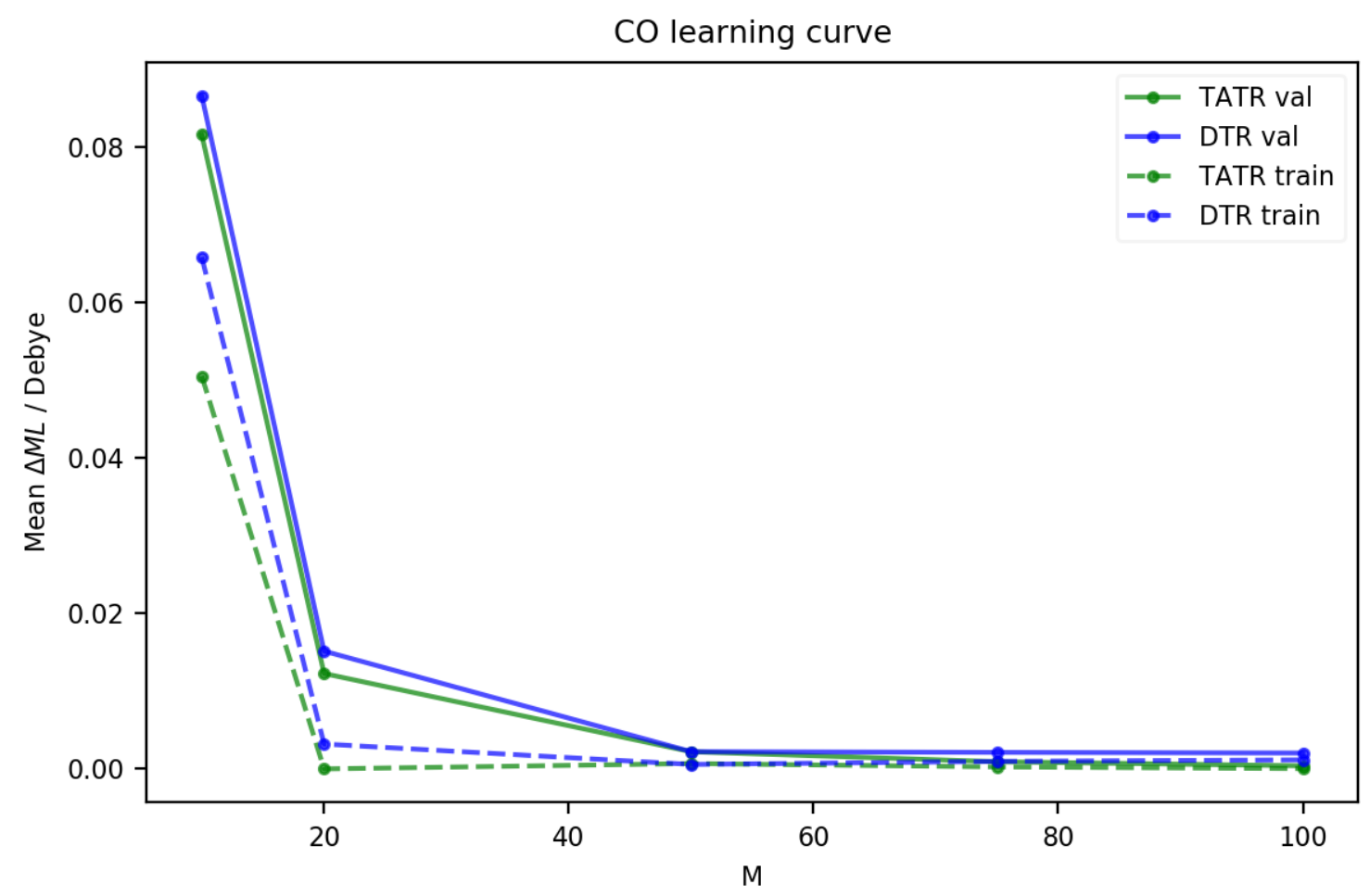

Figure S14: DTR and TATR learning curves for CO correlated dipole.

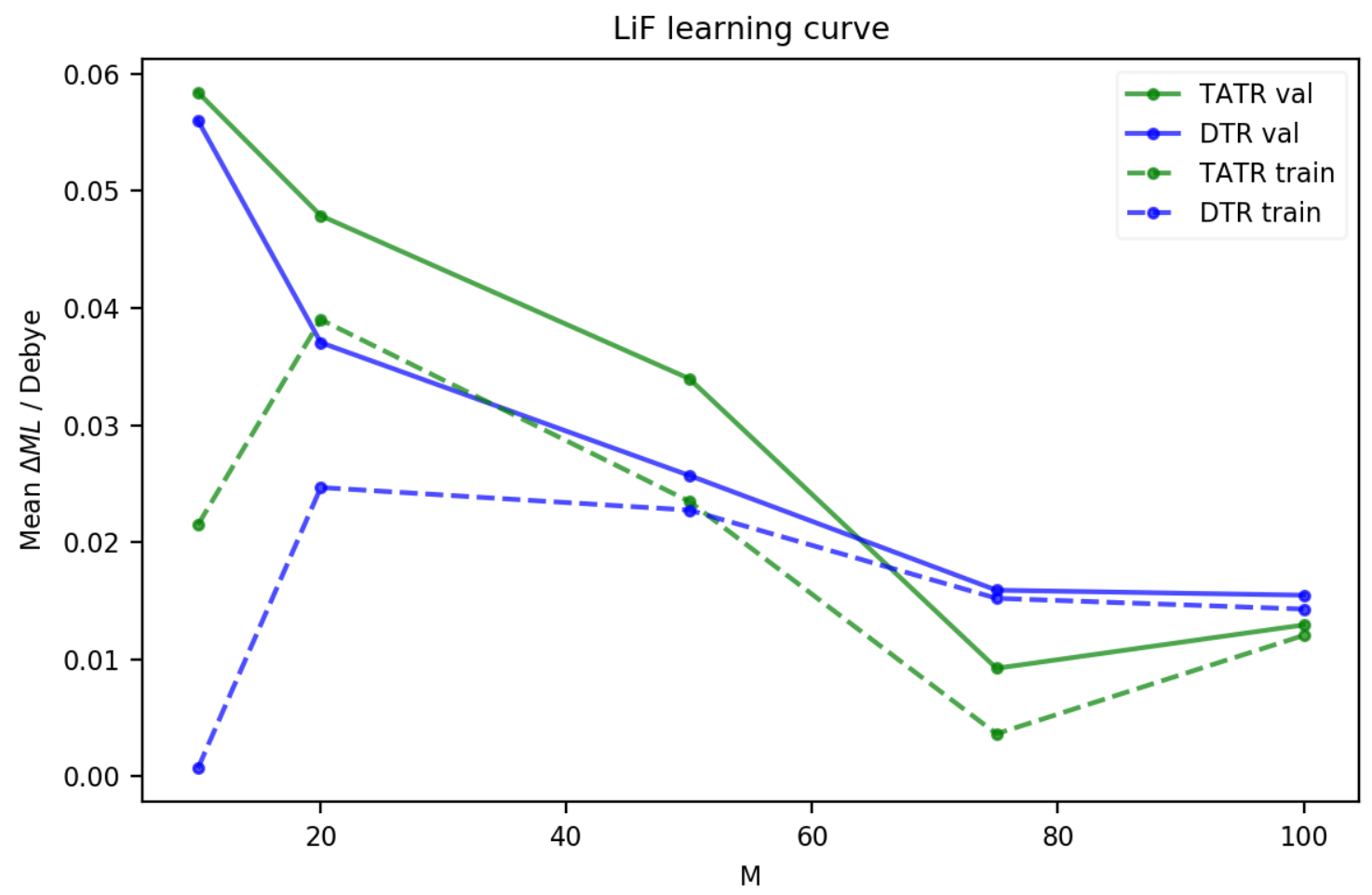

Figure S15: DTR and TATR learning curves for LiF correlated dipole. 


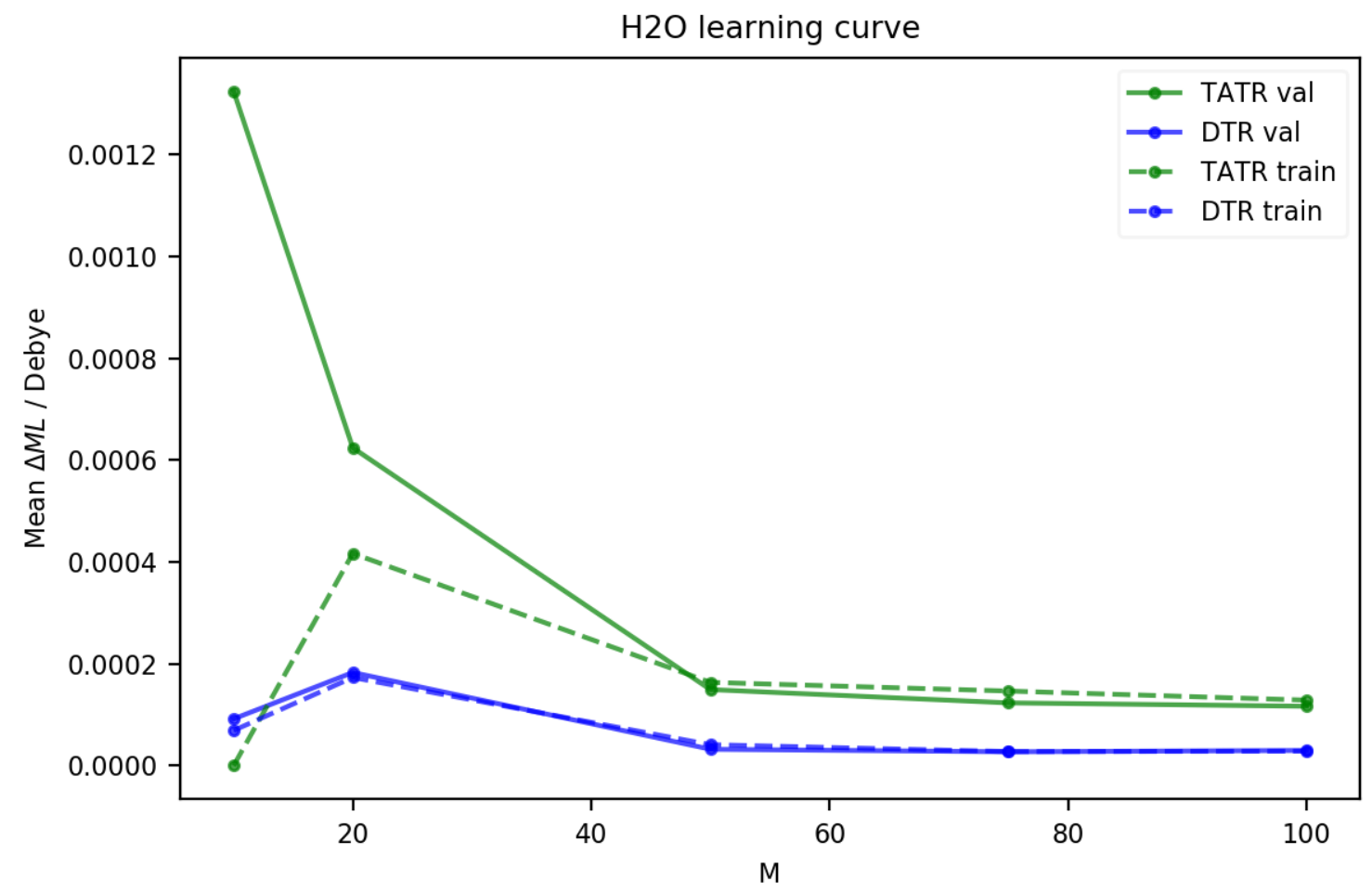

Figure S16: DTR and TATR learning curves for $\mathrm{H}_{2} \mathrm{O}$ correlated dipole.

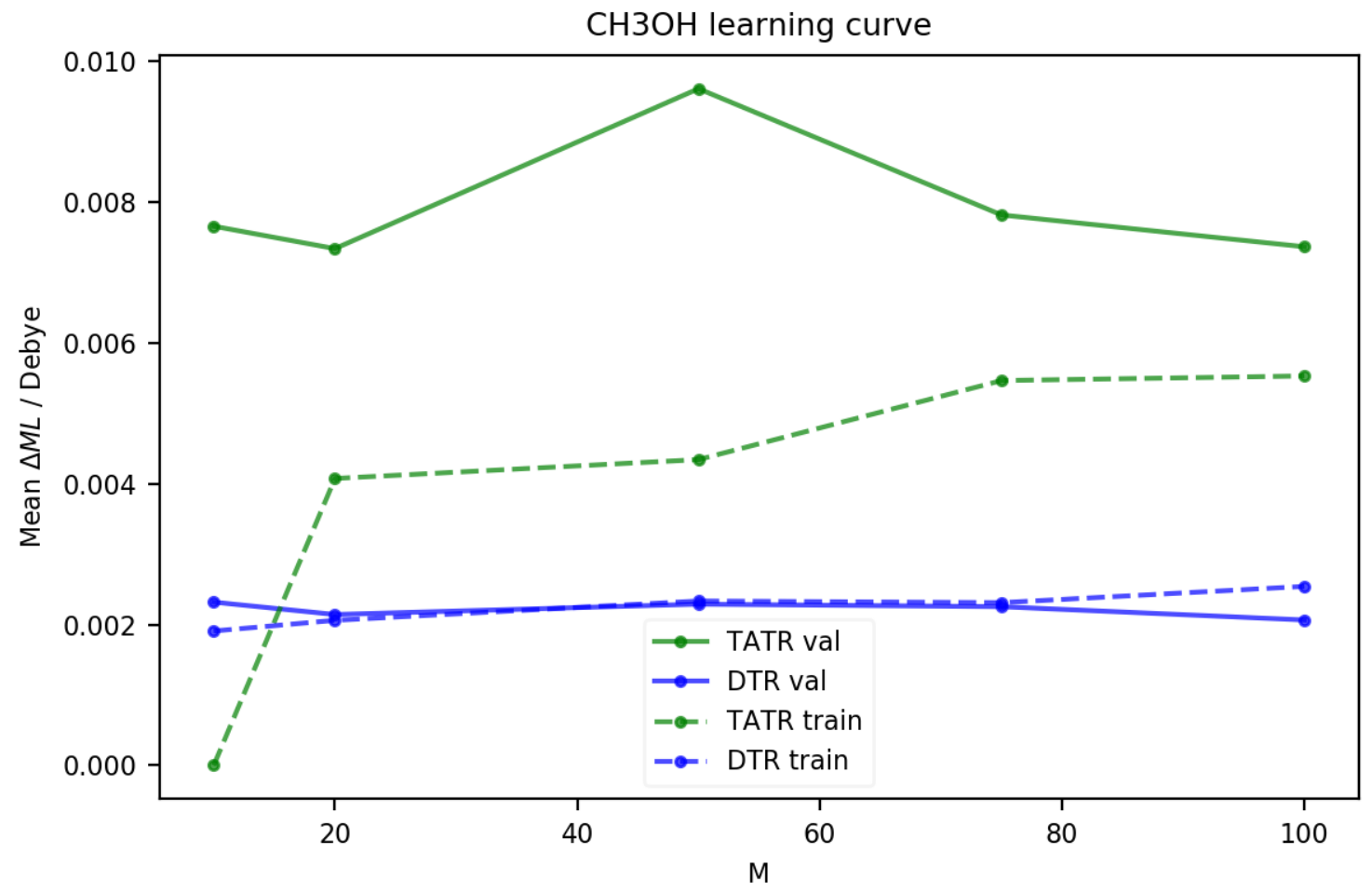

Figure S17: DTR and TATR learning curves for $\mathrm{CH}_{3} \mathrm{OH}$ correlated dipole. 


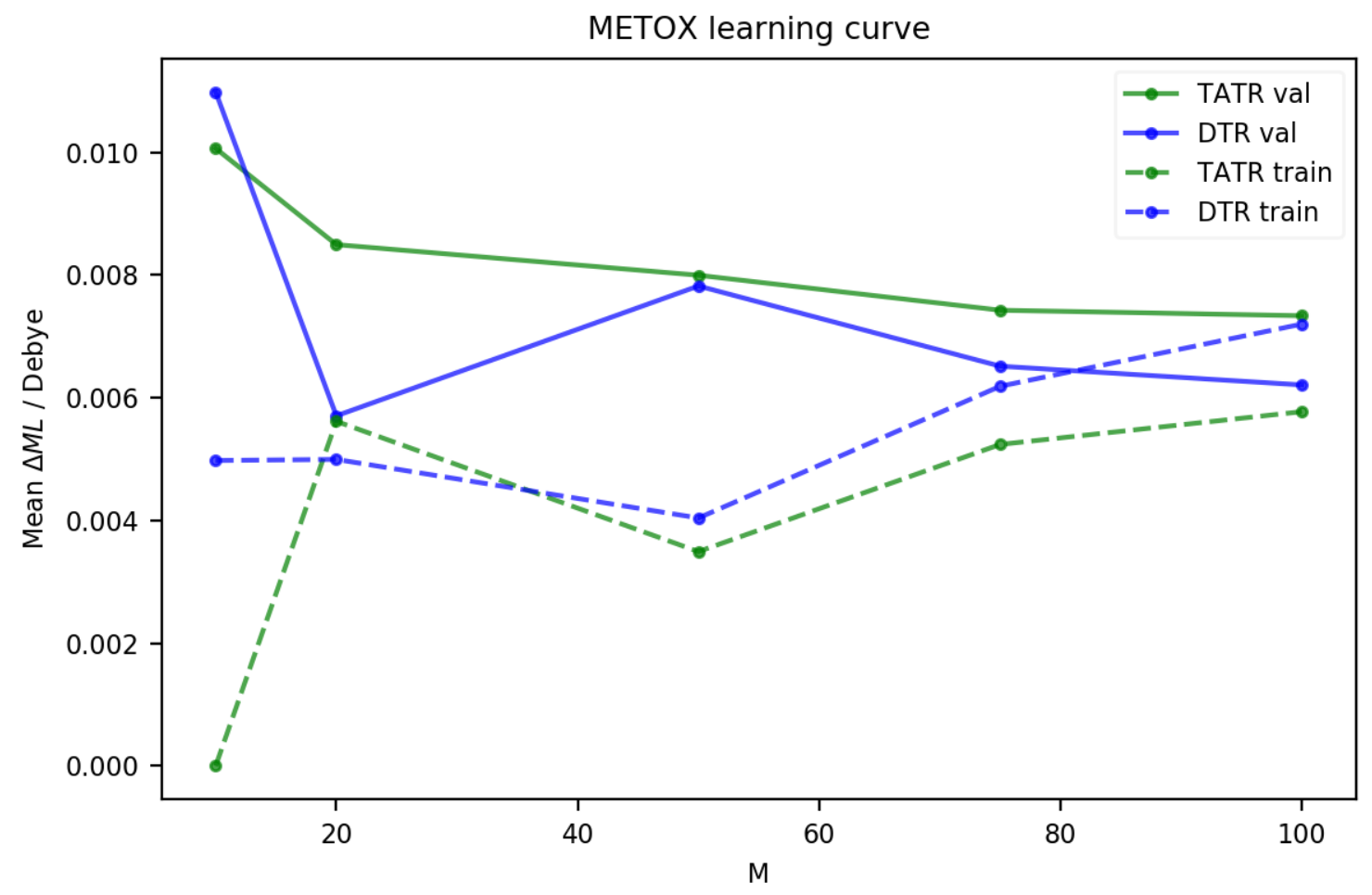

Figure S18: DTR and TATR learning curves for $(S)$-methyloxirane correlated dipole.

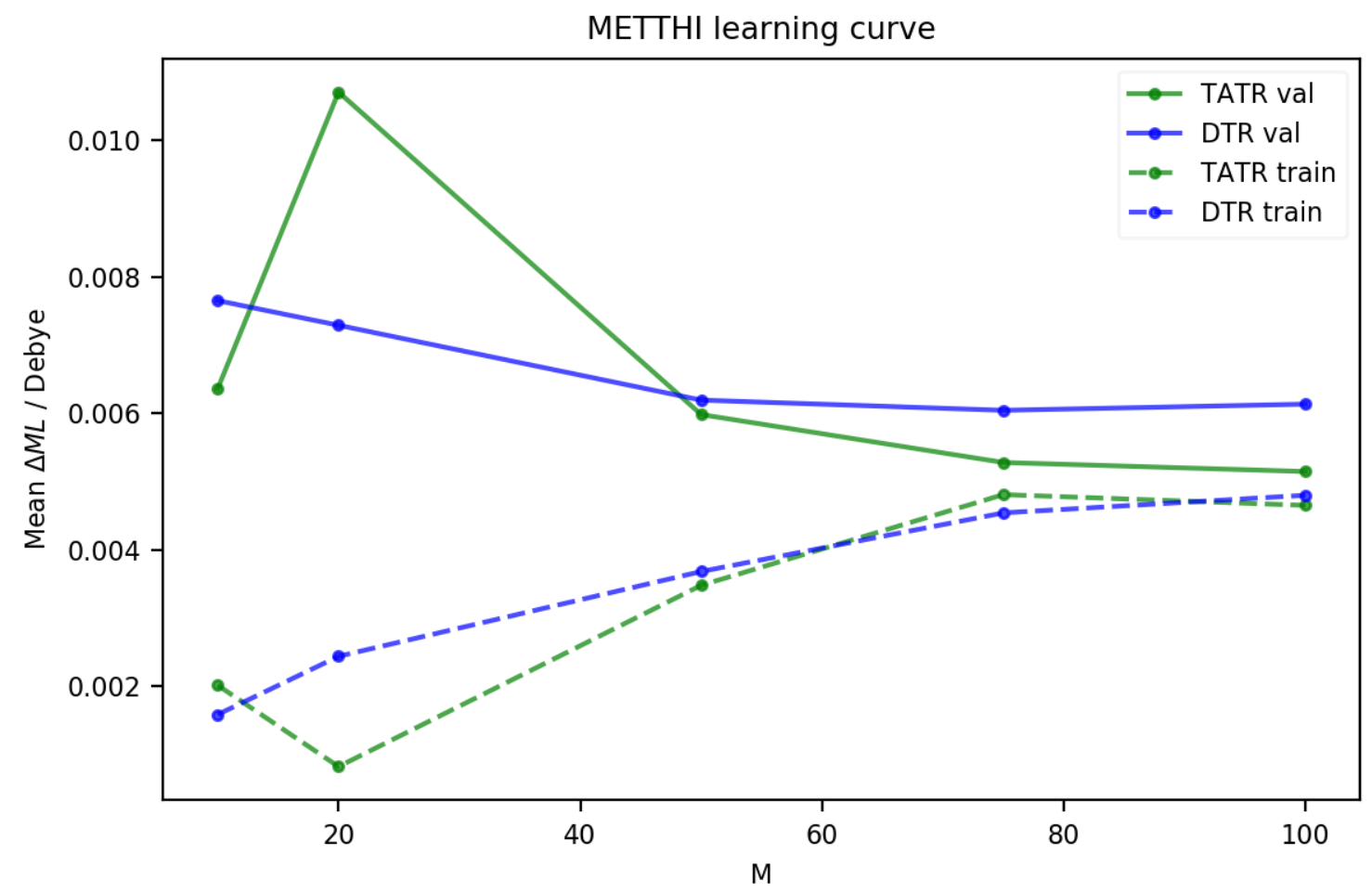

Figure S19: DTR and TATR learning curves for $(R)$-methylthiirane correlated dipole. 


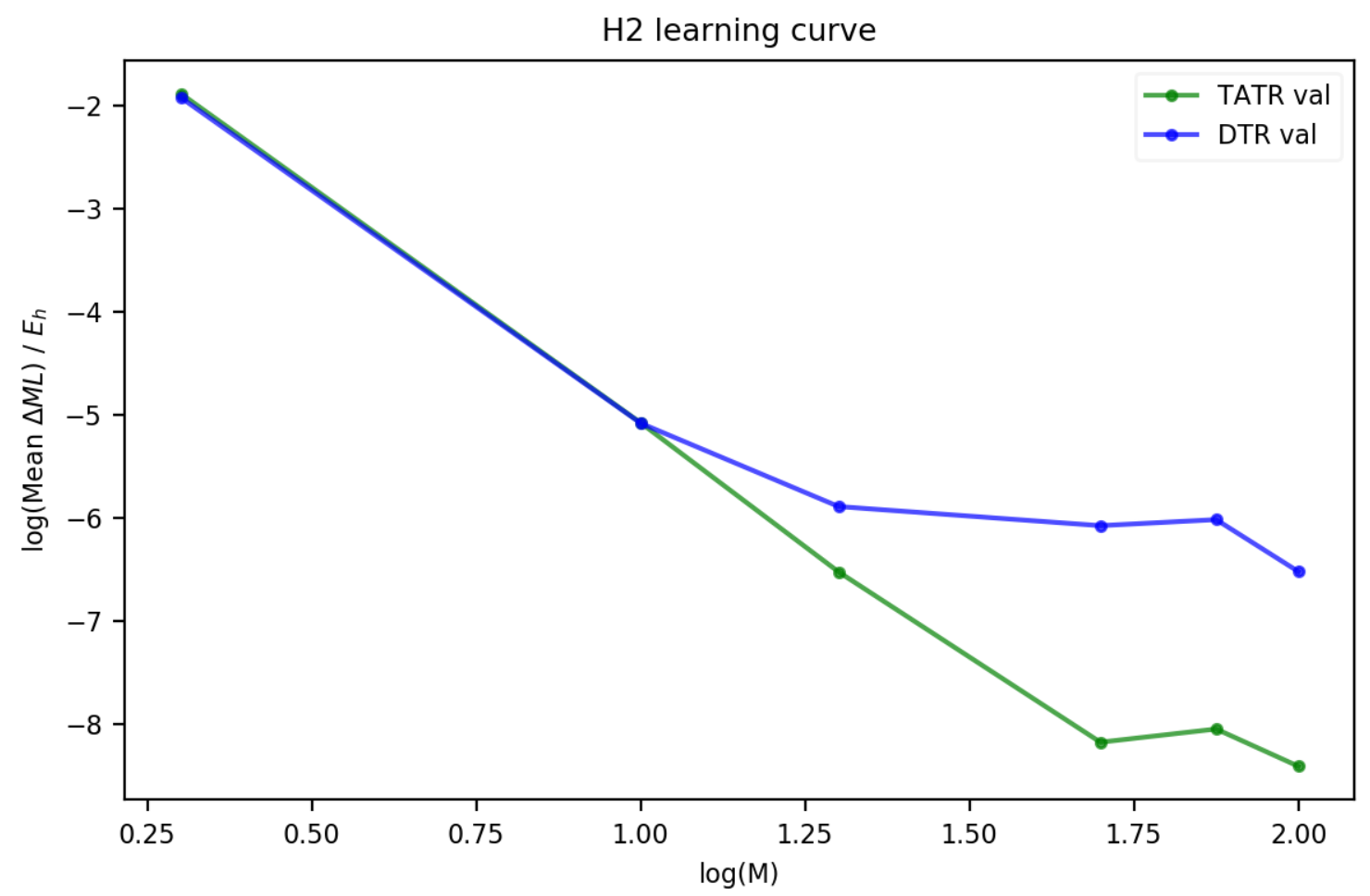

Figure S20: DTR and TATR validation curves for $\mathrm{H}_{2}$ correlation energy.

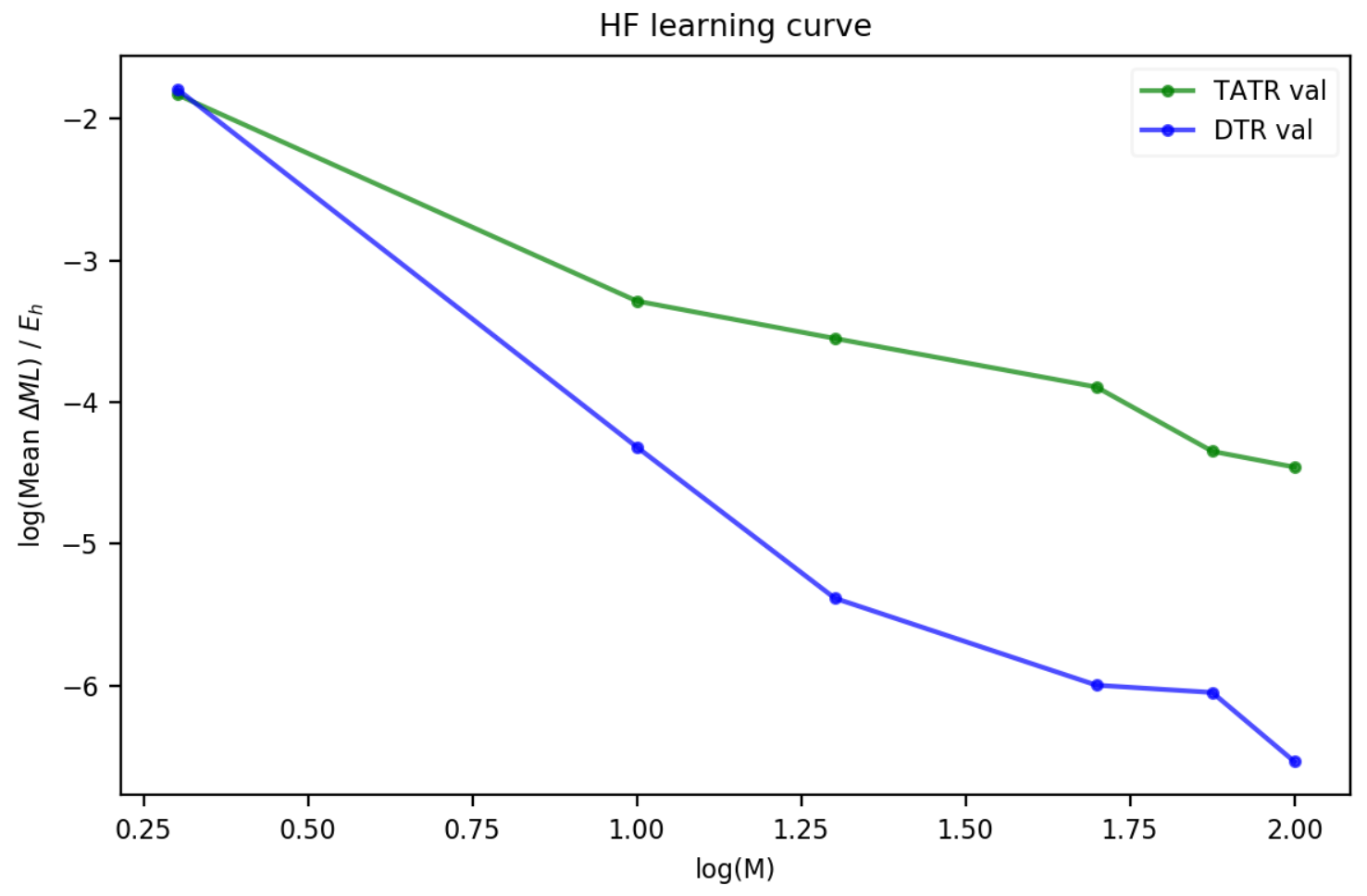

Figure S21: DTR and TATR validation curves for HF correlation energy. 


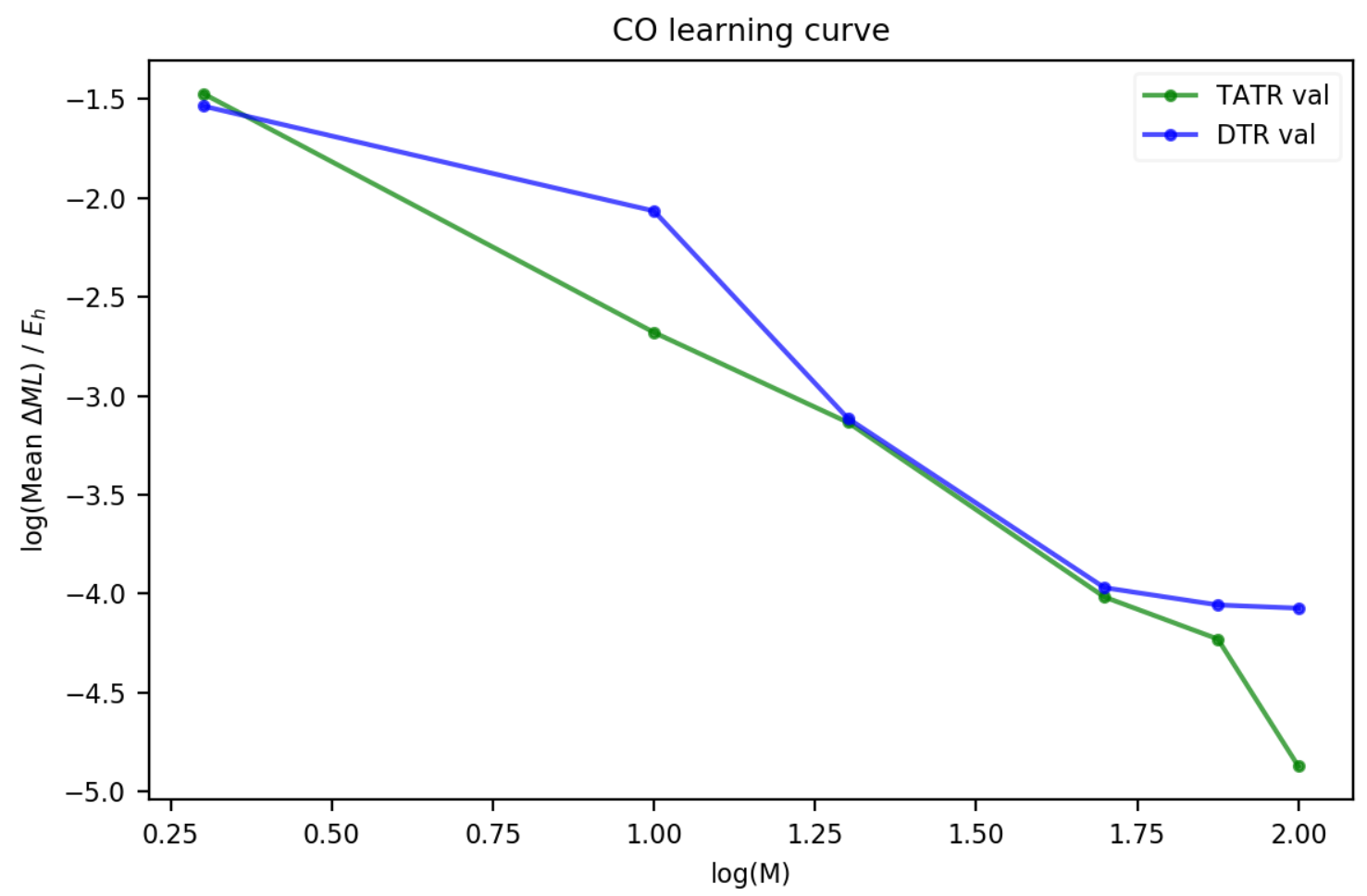

Figure S22: DTR and TATR validation curves for CO correlation energy.

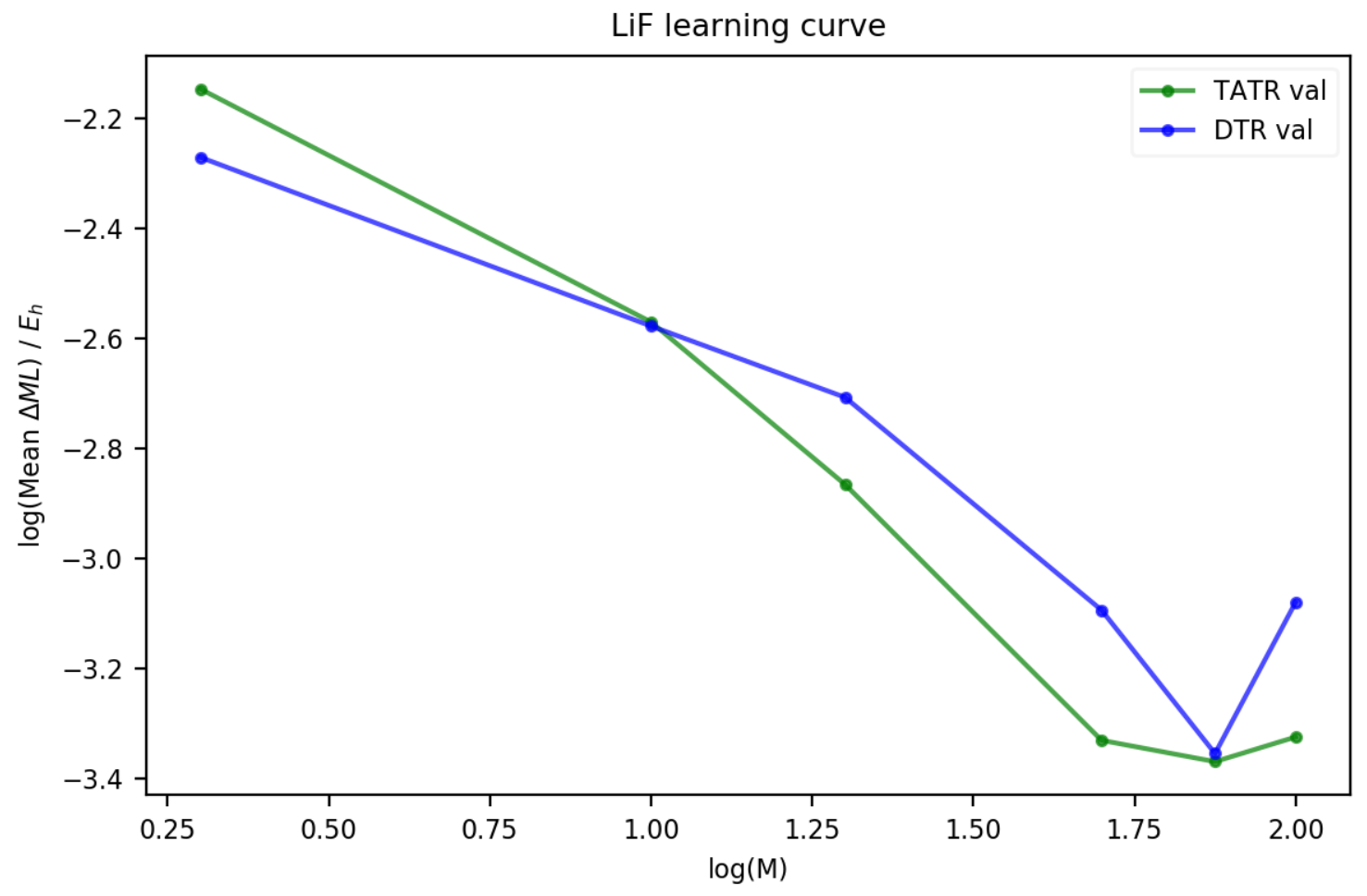

Figure S23: DTR and TATR validation curves for LiF correlation energy. 


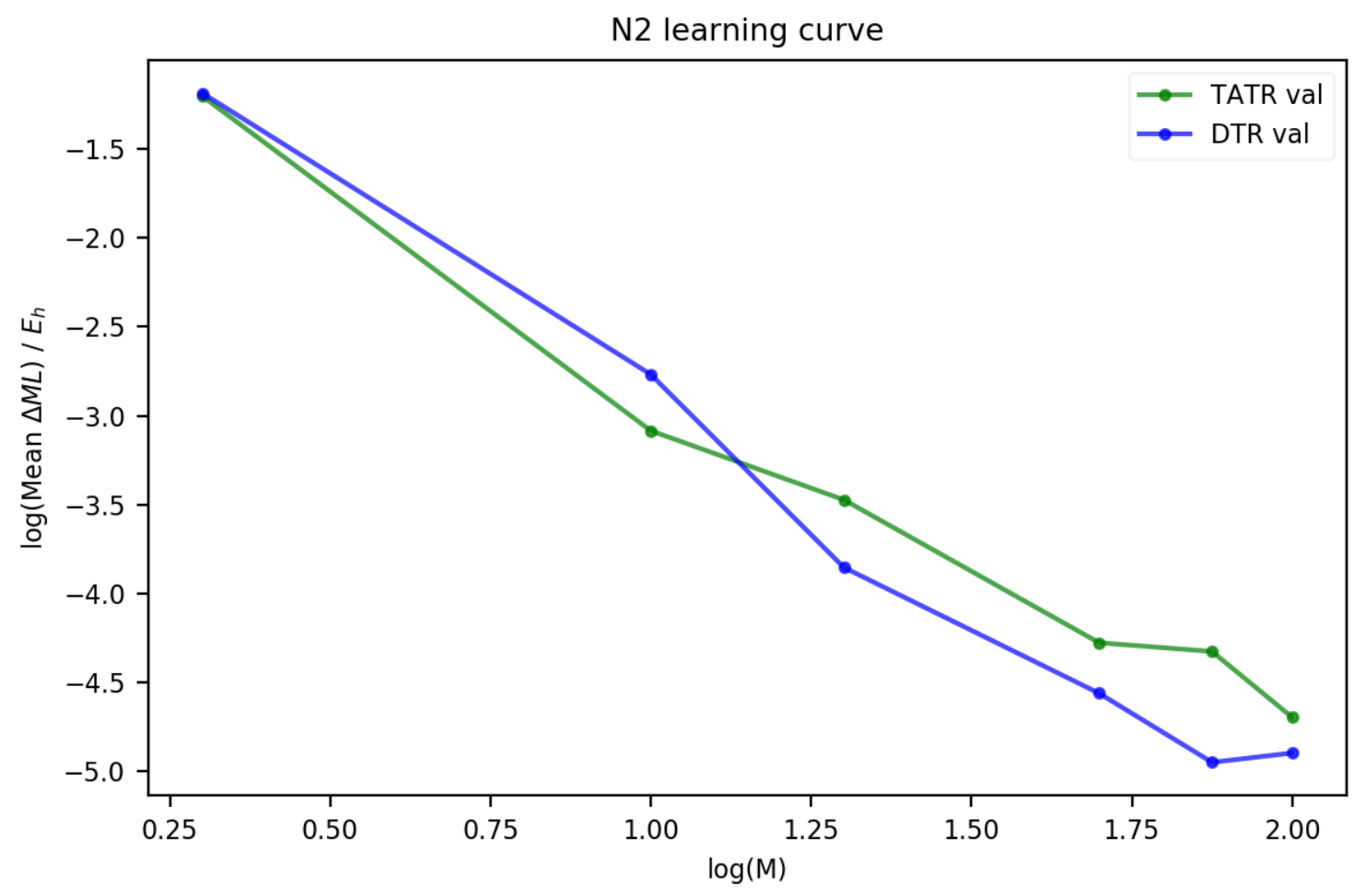

Figure S24: DTR and TATR validation curves for $\mathrm{N}_{2}$ correlation energy.

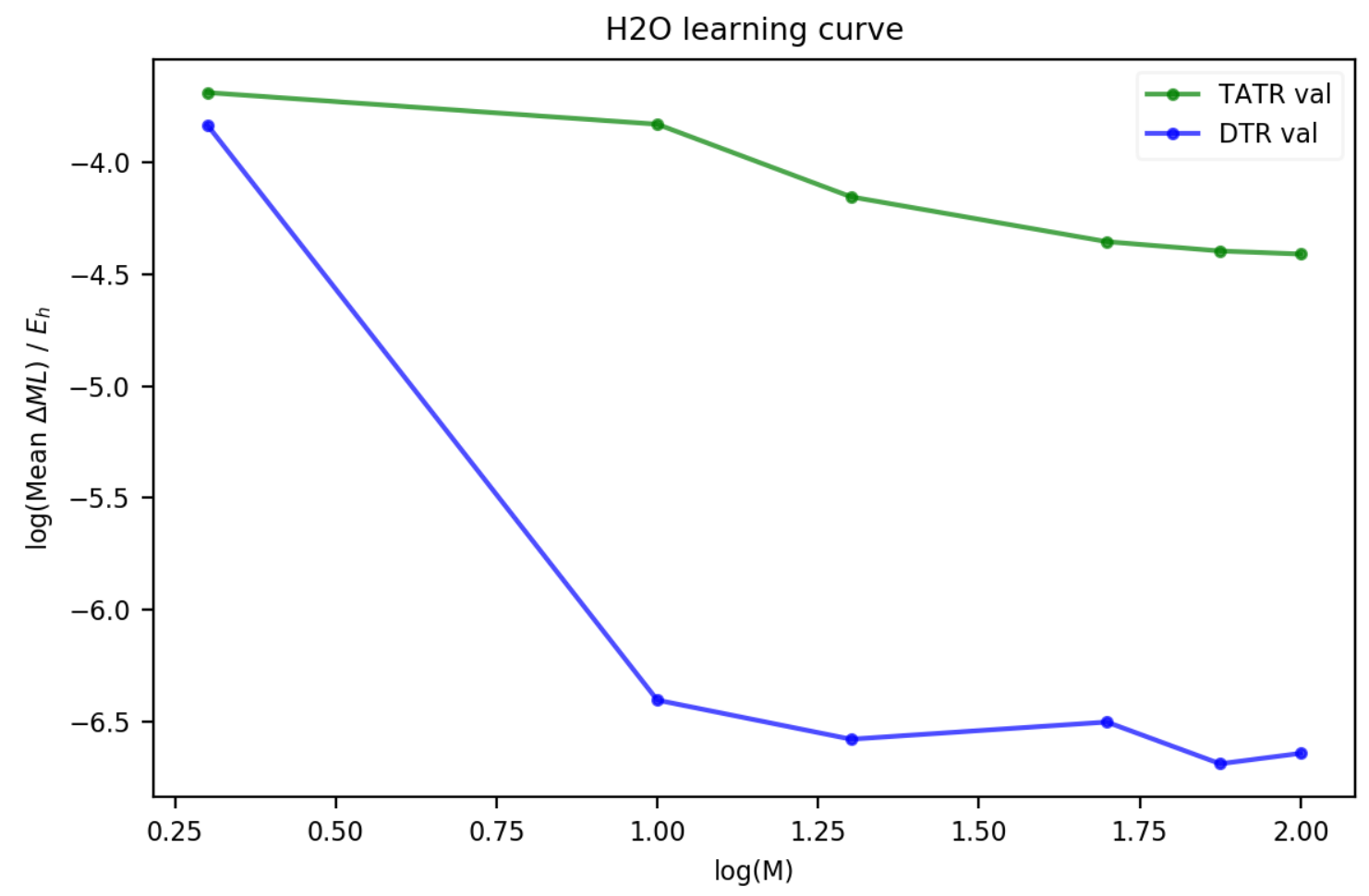

Figure S25: DTR and TATR validation curves for $\mathrm{H}_{2} \mathrm{O}$ correlation energy. 


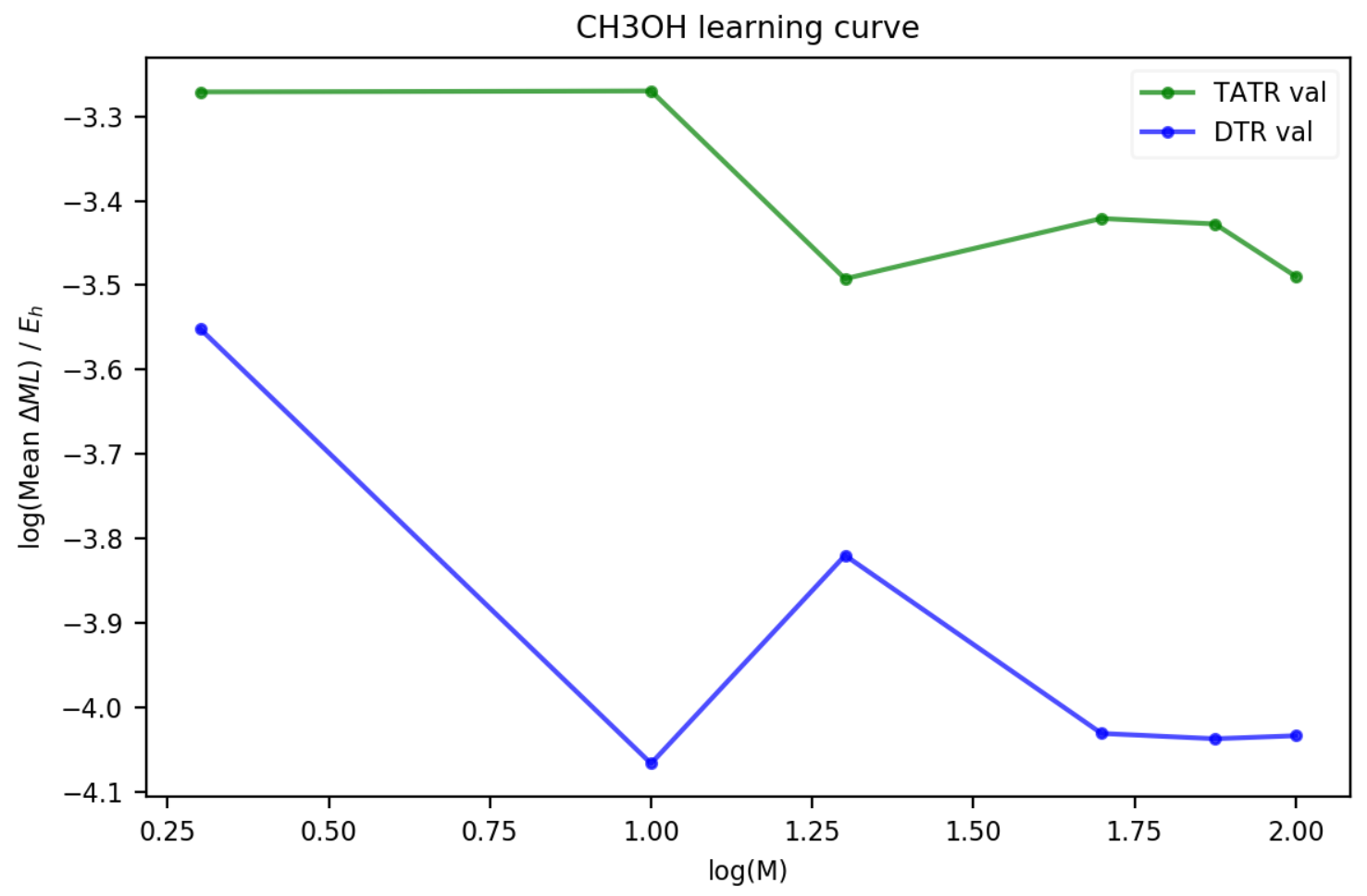

Figure S26: DTR and TATR validation curves for $\mathrm{CH}_{3} \mathrm{OH}$ correlation energy.

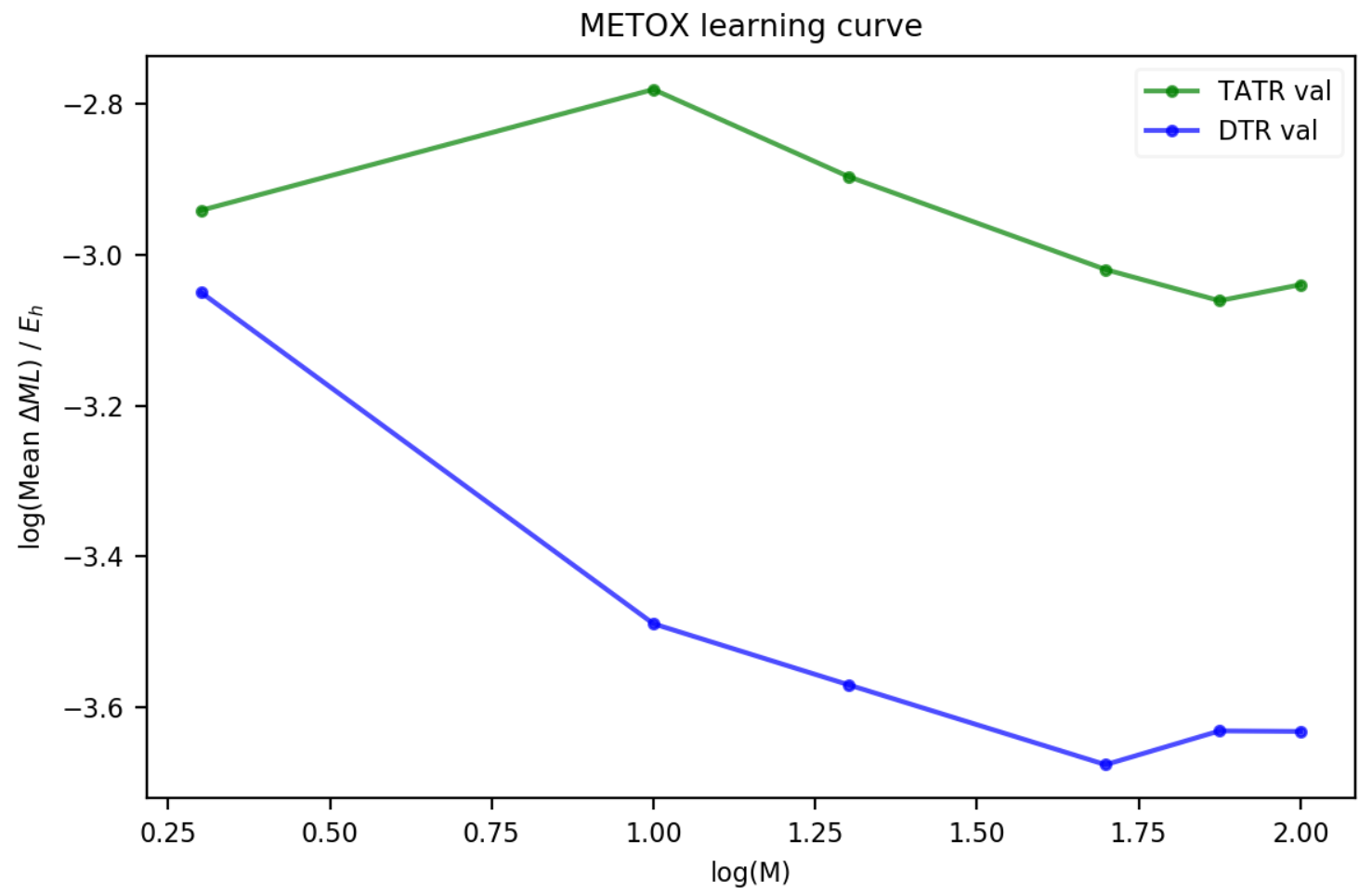

Figure S27: DTR and TATR validation curves for $(S)$-methyloxirane correlation energy. 


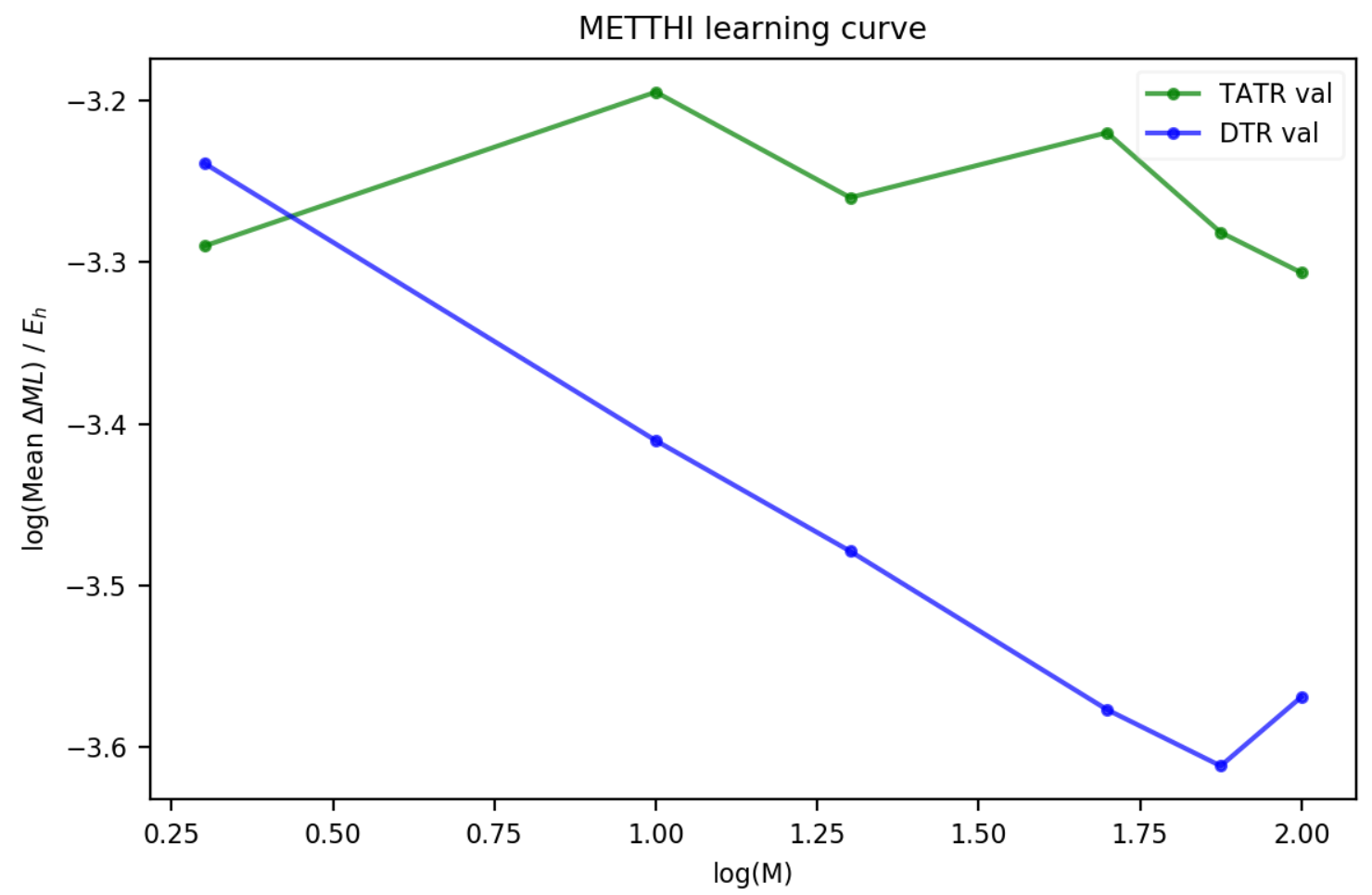

Figure S28: DTR and TATR validation curves for $(R)$-methylthiirane correlation energy.

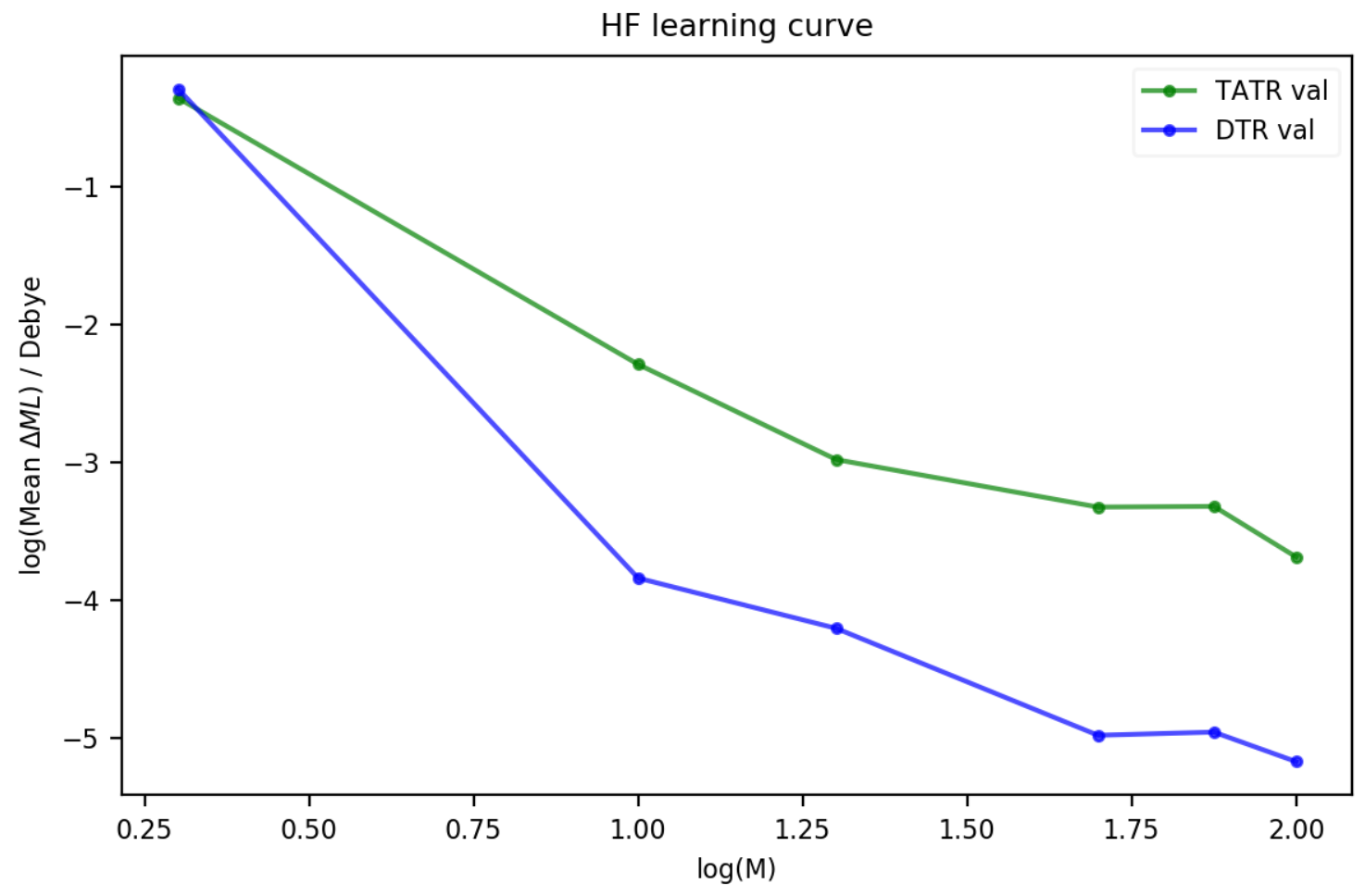

Figure S29: DTR and TATR validation curves for HF correlated dipole. 


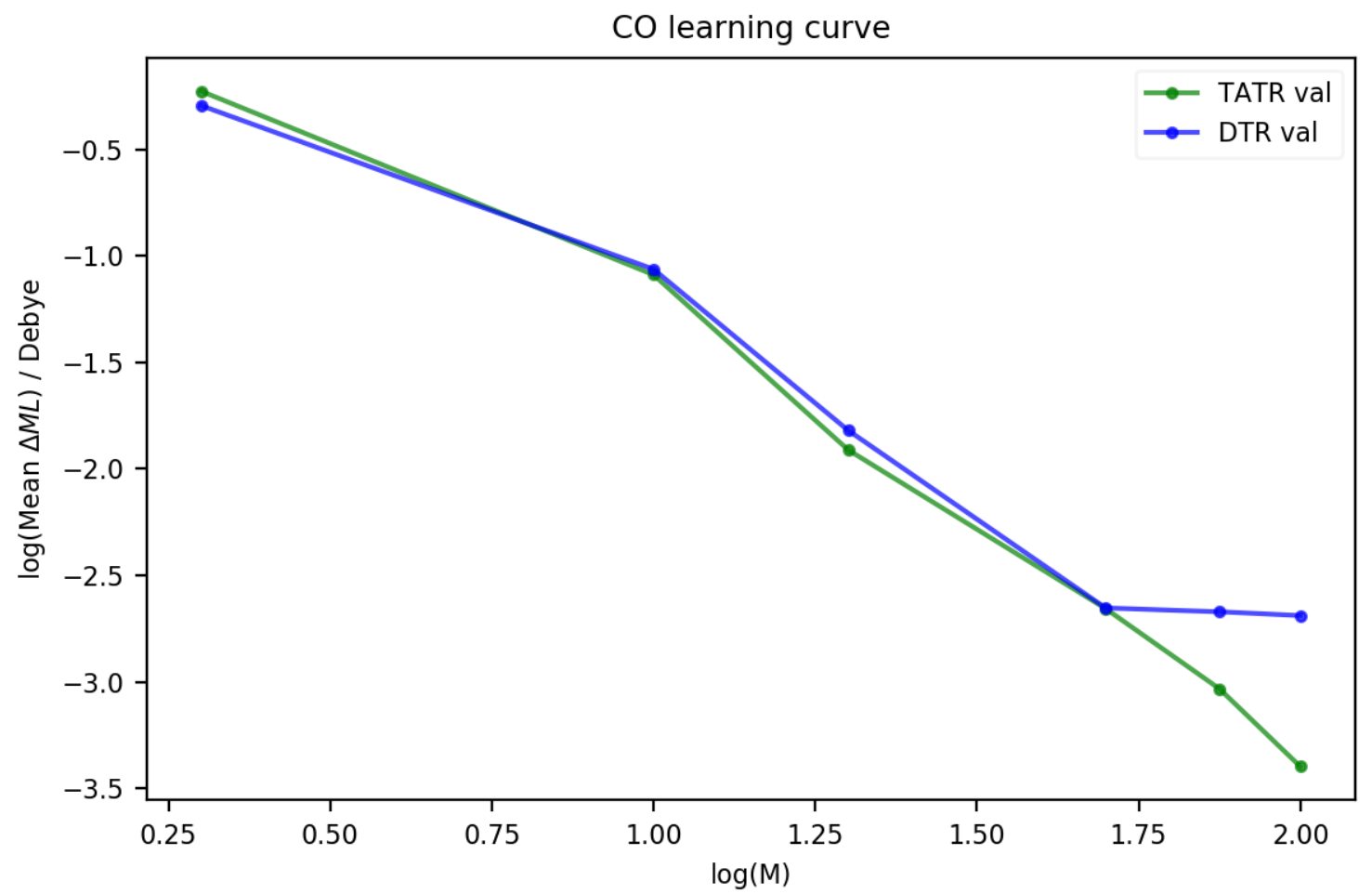

Figure S30: DTR and TATR validation curves for CO correlated dipole.

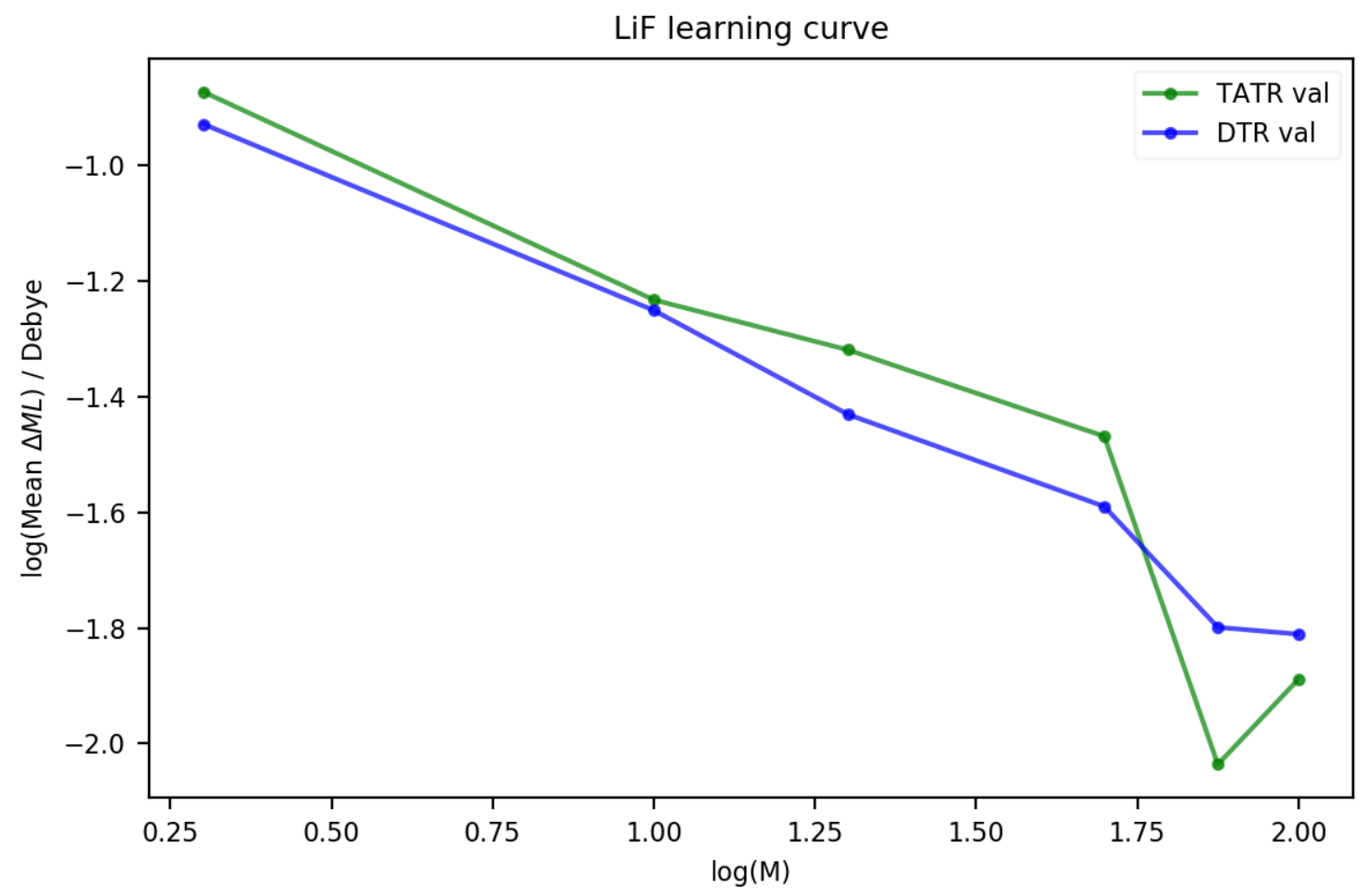

Figure S31: DTR and TATR validation curves for LiF correlated dipole. 


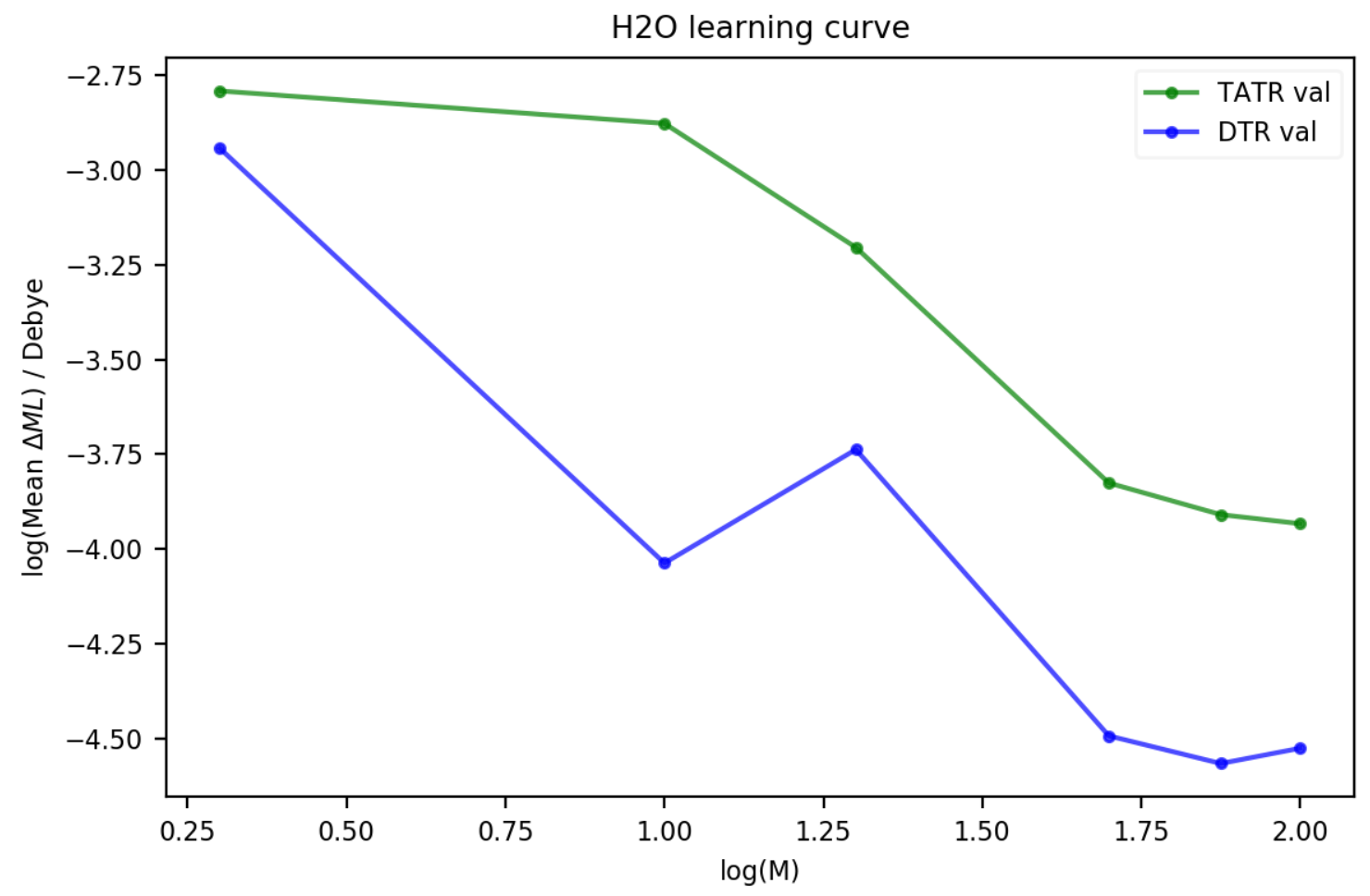

Figure S32: DTR and TATR validation curves for $\mathrm{H}_{2} \mathrm{O}$ correlated dipole.

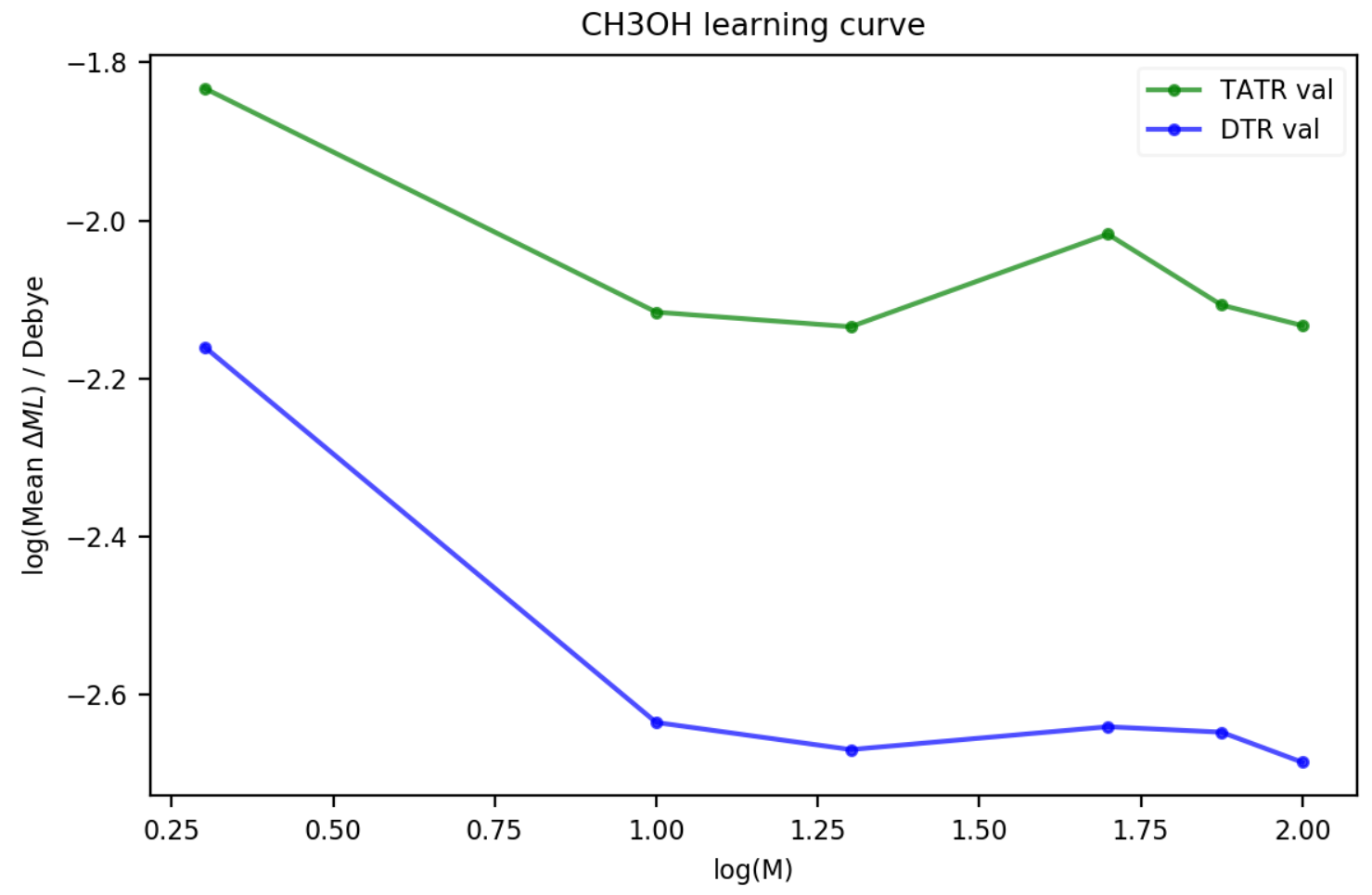

Figure S33: DTR and TATR validation curves for $\mathrm{CH}_{3} \mathrm{OH}$ correlated dipole. 


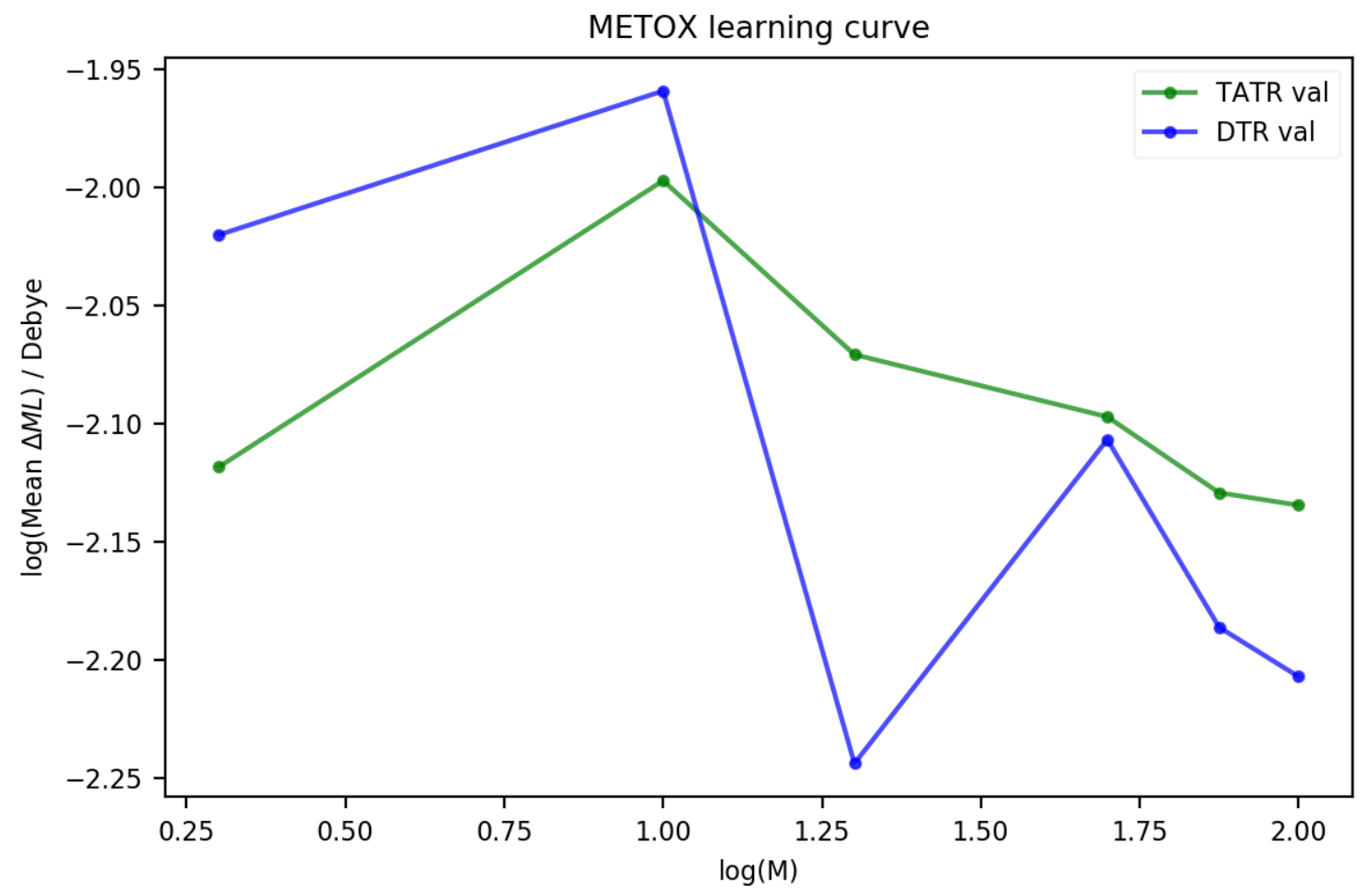

Figure S34: DTR and TATR validation curves for $(S)$-methyloxirane correlated dipole.

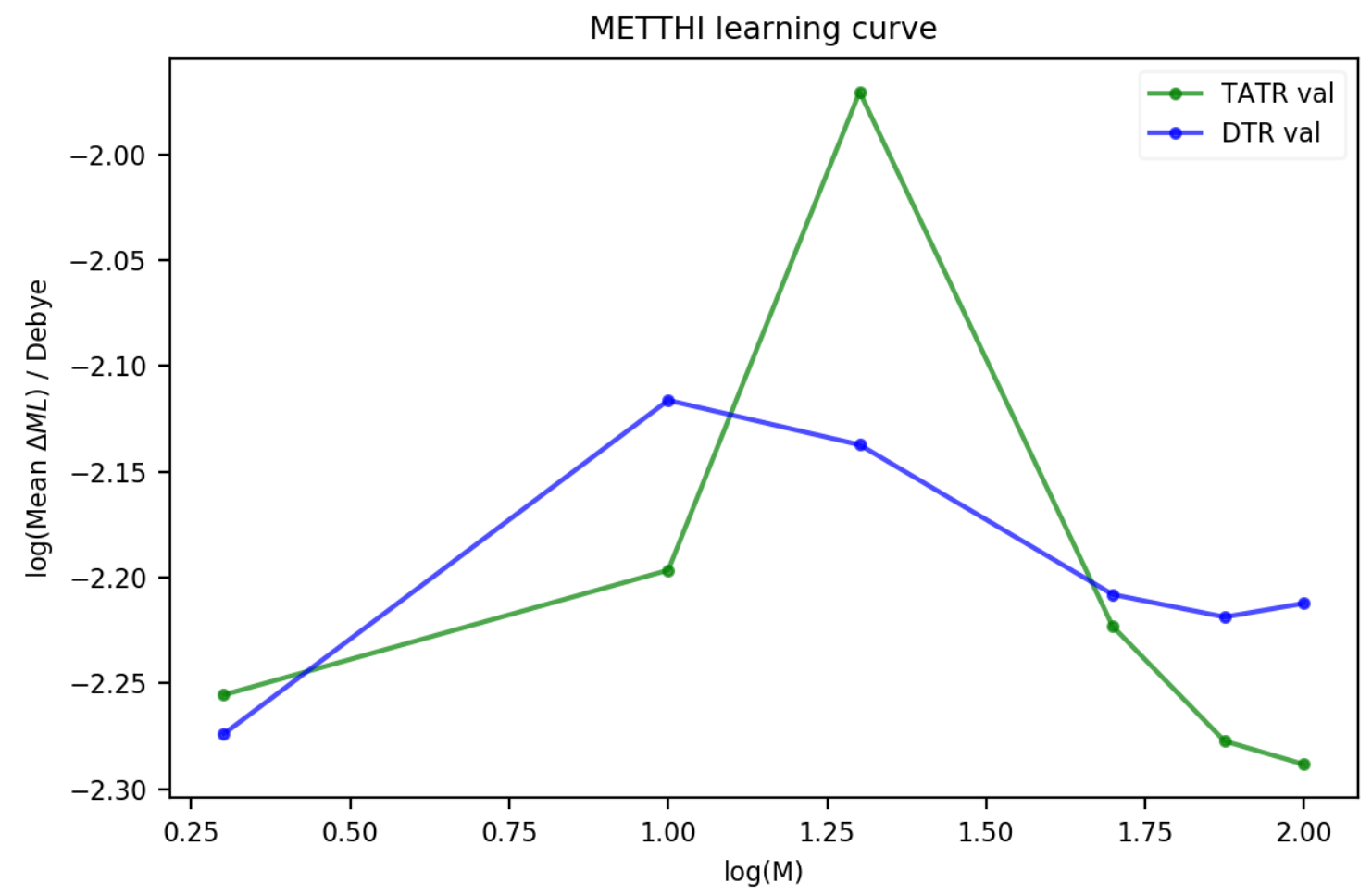

Figure S35: DTR and TATR validation curves for $(R)$-methylthiirane correlated dipole. 